\title{
Choice resolutions
}

\section{Domenico Cantone $^{1} \cdot$ Alfio Giarlotta $^{2}\left(\mathbb{D} \cdot\right.$ Stephen Watson $^{3}$}

Received: 30 July 2019 / Accepted: 11 September 2020 / Published online: 3 November 2020

(c) The Author(s) 2020

\begin{abstract}
We describe a process to compose and decompose choice behavior, called resolution. In the forward direction, resolutions amalgamate simple choices to create a complex one. In the backward direction, resolutions detect when and how a primitive choice can be deconstructed into smaller choices. A choice is resolvable if it is the resolution of smaller choices. Rationalizability, rationalizability by a preorder, and path independence are all preserved (backward and forward) by resolutions, whereas rationalizability by a weak order (equivalently, WARP) is not. We characterize resolvable choices, and show that resolvability generalizes WARP.
\end{abstract}

\section{Introduction}

The theory of revealed preferences pioneered by Samuelson (1938) postulates that preferences can be derived from choices: an agent's choice behavior is observed, and her preference structure is revealed from it. In this paper we present a theory of choice resolutions, which is inspired by the same principle of revealing a hidden attitude, but aims at inferring a different type of knowledge. In fact, the outcome of our analysis consists of an acquired information about a possible 'decomposability' of the selection process into simpler-typically more understandable-choices.

In real life, the process of choice naturally goes through several steps. Recent theories of bounded rationality provide different modelizations of this phenomenon: see, for instance, the approaches based on choice from menus-as in Dekel et al.

Alfio Giarlotta

giarlott@unict.it

Domenico Cantone

cantone@dmi.unict.it

Stephen Watson

watson@mathstat.yorku.ca

1 Department of Mathematics and Computer Science, University of Catania, Catania, Italy

2 Department of Economics and Business, University of Catania, Catania, Italy

3 Department of Mathematics and Statistics, York University, Toronto, Canada 
(2001), Gul and Pesendorfer (2001)—, sequential rationalization — as in Apesteguía and Ballester (2013), Au and Kawai (2011), García-Sanz and Alcantud (2015), Manzini and Mariotti (2007, (2012), Masatlioglu and Nakajima (2013), Tyson (2013)—, and simultaneous multi-rationalization - as in Cherepanov et al. (2013), Kalai et al. (2002). In a similar, but somehow orthogonal, way, choice resolutions aim at capturing the possibility to understand a possibly complicated choice behavior by deconstructing it in simpler choices at different stages.

Although the main use of choice resolutions is for deconstruction purposes- that is, going 'backward', starting from an observed choice behavior-, this technique can be also employed in the 'forward' direction, that is, to construct a choice out of simpler ones. However, in this paper we shall mostly concentrate on the backward' interpretation of resolutions, especially because of its relationship to theories of bounded rationality.

\subsection{Resolutions}

Our main goal is essentially descriptive, namely to obtain an accurate breakdown of a choice process into simultaneous/sequential stages. To eventually accomplish this ambitious task, here we deal with the simplest version of a decomposition procedure, called 'one-point resolution'. Nevertheless, we wish to clarify from the outset that this limitation is only apparent: in fact, a complete decomposition of a choice in elementary steps can always be obtained by a sequence of one-point resolutions.

The idea of a one-point resolution is to deconstruct/reconstruct a primitive choice behavior using the following ingredients: (1) two disjoint choices, the 'base' and the 'fiber', defined on smaller ground sets; (2) a selected item in the base set, which is 'blown-up' into the fiber choice; and (3) an assumption of independence of the base and the fiber. More formally, we identify a base choice $\operatorname{space}^{1}\left(X, c_{X}\right)$ and a special item $x \in X$, and then resolve $x$ into a fiber choice space $\left(Y, c_{Y}\right)$, thus obtaining a larger choice space

$$
\left(Z, c_{Z}\right)=\left(X, c_{X}\right) \oplus_{x}\left(Y, c_{Y}\right)
$$

Here the set $Z$ is formed by substituting the item $x$ by the set $Y$, whereas the choice $c_{Z}$ is a meaningful amalgamation of the choices $c_{X}$ and $c_{Y}$. The pair $\left(Z, c_{Z}\right)$ is called the one-point resolution of $\left(X, c_{X}\right)$ at $x$ into $\left(Y, c_{Y}\right)$. Figure 1 gives an intuitive representation of such a process: the dotted lines suggest the idea of taking a magnifying glass, and looking at the item $x$ in $\left(X, c_{X}\right)$ as being itself a menu with its own choice structure $\left(Y, c_{Y}\right)$.

Example 1 (Carte Blanche) The CEO of a corporation gives full authority to the Vice-President (VP) of the Research and Development (R\&D) Department within

\footnotetext{
${ }^{1}$ A choice space is a pair $\left(X, c_{X}\right)$, where $X$ is a nonempty set, and $c_{X}: \Omega_{X} \rightarrow \Omega_{X}$ is a map defined on a nonempty family $\Omega_{X}$ of nonempty subsets of $X$ with the property that $c(A) \subseteq A$ for any $A \in \Omega_{X}$. In this paper, we shall only address the case $\Omega_{X}:=2^{X} \backslash\{\emptyset\}$, that is, the choice domain is the collection of all nonempty subsets of $X$.
} 
an allotted budget: the goal is to develop new techniques to acquire market quotas, currently owned by competitors. Thus, all choices on the projects/investments of the R\&D Department are made by its VP. In this model, the choices made by the CEO are encoded by $\left(X, c_{X}\right)$, the item $x$ is represented by the investments concerning the R\&D Department, and $x$ is resolved into the choices effectively made by the R\&D Department, encoded by $\left(Y, c_{Y}\right)$.

The operation of one-point resolution naturally extends in two directions:

(i) vertically, by 'sequentially' repeating the decomposition process any (possibly infinite) number of times for distinct base and fiber spaces;

(ii) horizontally, by 'simultaneously' resolving several (possibly all) items of the base space into fiber spaces.

The combination of vertical/horizontal resolutions yields a resolution tree, whose branches exhibit all delegations of independent tasks of an observed choice behavior. $^{2}$

Applications of resolutions are possible in several scenarios, e.g., corporate structures, investment portfolios, hiring process with limited budget. For instance, consider again the case of a corporation, whose complex organization induces the CEO to partially delegate decision authority to VPs of departments (which, in turn, have their own hierarchical structure). Then detecting the existence of fully autonomous units in the corporation by just looking at its choice behavior on projects may have a high strategic impact in the decision-making process of its competitors. In this context, the next example suggests how resolutions may apply in an 'unfair' market competition.

Example 2 (Divide et Impera, alias Decompose and Bribe) Assume a corporation $A$ wants to (re)gain competitiveness over another corporation $B$. To accomplish this goal at any cost, the CEO of $A$ assembles a team of analysts. By carefully studying the investments of $B$, this team establishes that the decisions of the R\&D Department of $B$ are fully independent of the CEO of $B$. (For instance, imagine a situation in which all technical choices related to research are too novel and complicated to be judged by the CEO.) As a consequence, the CEO of $A$ contacts the VP of the R\&D Department of $B$, and bribes him to make research and development of $B$ go to a dead end.

In a different direction, resolutions are useful for obtaining a compact representation of a choice behavior in terms of simpler choices. The next example illustrates this point.

\footnotetext{
${ }^{2}$ Needless to say, the possibility to have a stochastic dependence of the fibers from the base would make our model more realistic and powerful. However, dropping the assumption of independence requires a more sophisticated approach, so here we restrict our analysis to the simple case of independent choices.
} 
Fig. 1 A one-point resolution $\left(Z, c_{Z}\right)=\left(X, c_{X}\right) \oplus_{x}\left(Y, c_{Y}\right)$

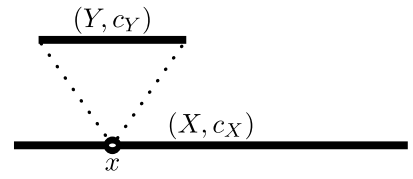

Example 3 (Gemma's choice) On Sundays, Gemma and her family have lunch at the same restaurant. Gemma only likes a few dishes, namely pizza $(p)$, chips $(c)$, sea bass $(s)$, and tuna $(t)$. She chooses as follows (selected items are underlined):

$$
p \underline{c s t}, p \underline{c s}, p \underline{c t}, \underline{p s t}, \underline{c s t}, \underline{p c}, \underline{p s}, \underline{p t}, \underline{c s}, \underline{c t}, \underline{s t} .
$$

Thus, for instance, pcst says that Gemma selects sea bass and chips even if pizza and tuna are also available in addition to them. The core of Gemma's preferences is explained by a linear sequence of strict preferences over menus, namely $\{c, s\}>\{c, t\}>\{p\}>\{s\}>\{t\}$, and everything else follows from it. Despite the simplicity of this choice, one-point resolutions do possess explanatory power also in this case. To illustrate the utility of the resolution model, let $f$ (fish) be a 'new' item, and proceed as follows:

- $\left(X, c_{X}\right)$ is the base choice, where $X=\{p, c, f\}$, and $c_{X}$ is given by $p \underline{c f}, p c, p f, \underline{f} f$

- $f$ is the base point in $X$;

- $\left(Y, c_{Y}\right)$ is the fiber choice, where $Y=\{s, t\}$, and $c_{Y}$ is given by $\underline{s} t$.

Then the choice (1) can be 'decomposed' as follows: ${ }^{3}$

$$
p \underline{c} \underline{f}, \underline{p c}, \underline{p} f, \underline{c} \underline{f} \oplus_{f} \underline{s} t
$$

In other words, Gemma's behavior has two representations, namely (1) and (2): the first is 'direct', and explicitly describes her tastes on each available menu; the second is 'indirect', and uses two simpler selections to describe her tastes. It is apparent that (2) is a more compact-and, possibly, more effective-description than (1).

\subsection{Three questions}

A first task of this paper is to examine the 'rationality' features of the resolution model. Recall that a choice is rationalizable whenever there is a binary relation such that the choice behavior is obtained by the maximization of this relation. Thus, it is natural to ask whether resolutions preserve (back and forth) the axioms of choice consistency that are related to rationalization, such as Samuelson's (1938) WARP (Weak Axiom of Revealed Preference), Chernoff's (1954) property $(\alpha)$, and Sen's (1971) property $(\gamma)$, that is,

\footnotetext{
${ }^{3}$ See Example 8 in Sect. 4.2 for all technical details.
} 
Question 1: If an axiom of consistency holds for both the base choice and the fiber choice, does it also hold for the resolved choice (forward preservation)? What about the converse (backward preservation)?

We show that the answer to the above question is positive-both forward and backward-for most of these properties. In particular, a resolved choice is rationalizable if and only if the base choice and the fiber choice are both rationalizable. However, one-point resolutions do not typically preserve (forward) the rationality features encoded by WARP. In other words, it usually happens that even if the base choice and the fiber choice are both rationalizable by a total preorder (reflexive, transitive, and complete), the transitivity of the revealed preference is lost in passing to the resolved choice.

From an algorithmic point of view, resolutions may provide an alternative way to test the rationalizability of a choice. This is linked to the resolvability of a choice, that is, the possibility to understand a given choice as the resolution of two simpler choices. In fact, instead of testing directly that the original choice satisfies the two consistency properties $(\alpha)$ and $(\gamma)$-which, as it is well-known, characterize rationalizability-, one may first test whether a choice is resolvable, and only then whether properties $(\alpha)$ and $(\gamma)$ hold for these simpler choices. Since the above procedure relies on an algorithm to test the resolvability of a choice, we also ask

Question 2: Can we effectively detect whether a choice can be explained as the resolution of two simpler choices?

In this paper, we provide a constructive answer to this question, characterizing resolvable choices by the existence of a shrinkable menu: this is a subset of the ground set such that an external observer cannot discern its choice structure from outside the menu itself.

For descriptive purposes, in this paper we provide a taxonomy of resolutions for small ground sets, determining (up to isomorphisms) all choices on a three-element set that are resolvable. It turns out that these choices are also rationalizable. This enlightening fact suggests that the two notions of choice rationalizability and choice resolvability may be linked to each other. Thus, we raise a last query:

Question 3: Is there any relationship between rationalizability and resolvability?

Question 3 may look redundant. Indeed, on the one hand, rationalizability is a 'global' property, which reveals an underlying preference structure (in fact, the maximization of a binary relation explains choice behavior). On the other hand, resolvability is a 'local' property, which reveals the possibility to deconstruct the primitive choice behavior at a point (in fact, a magnifying glass is applied to an item of the base space). Maybe contrary to expectations, there is a striking connection: in fact, resolvability generalizes WARP. 


\subsection{Relation with literature}

From a methodological point of view, the technical features of our model link it to the literature in mathematics. In fact, the analysis developed in this paper is inspired by that of a topological resolution, originally introduced by Fedorčuk, V. V. (1968), and then extensively studied by Watson (1992). This elegant mathematical notion has proven to be very useful in the field of set-theoretic topology, providing a common background for many seemingly different topological spaces.

From a similar point of view, our model is also reminiscent of a well-known technique in graph theory: see the pioneering paper of Gallai (1967), as well as the follow-up literature on modular decompositions of graphs and more general structures. Graph decomposition has proven relevant also in economics: see, e.g., Moulin (1986) for a model of voting that employs these types of tools. Furthermore, the notion of composition of binary relations, as given in Bang-Jensen and Gutin (2001, p. 8), is a particular case of choice resolutions.

From a different point of view, since we aim at detecting some facts underlying a choice behavior, the topic of this paper is linked to the literature on revealed preferences and bounded rationality. We shall mention several references of this kind along the way. Here we only recall some classical papers on the rationalizability of a choice, namely Samuelson (1938), Chernoff (1954), Arrow (1959), Sen (1971), and Plott (1973): their results will be fundamental in showing that most features of rationality are fully preserved by resolutions.

\subsection{Organization of the paper}

Section 2 defines one-point resolutions and shows its full generality for decomposition purposes (Theorem 1). Section 3 answers Question 1, showing that rationalizability, rationalizability by a preorder, path independence, and rationalizability by two sequential criteria are preserved back and forth by resolutions (Theorems 2, 3, and 4); however, rationalizability by a total preorder is only preserved in special cases (Theorem 5). Section 4 answers Question 2, giving a descriptive characterization of resolutions by means of the notion of shrinkable menu (Theorem 6); it also shows that shrinkability is a generalization of the notion of 'indiscernibility' (Theorem 7). Section 5 partially answers Question 3, showing that any nontrivial choice satisfying WARP is resolvable (Theorem 8). Section 6 suggests future directions of research. All proofs are in the Appendix.

\section{Resolutions}

In this section we introduce our model, prove its generality, give some examples, and describe possible interpretations. To start, we provide basic terminology.

In what follows, $X$ is a nonempty set of alternatives, and $\Omega_{X}=2^{X} \backslash\{\emptyset\}$ is the family of all nonempty subsets of $X$. A choice correspondence on $X$ is a map 
$c: \Omega_{X} \rightarrow \Omega_{X}$ such that $c(A) \subseteq A$ for all $A \in \Omega_{X}$; we call $X$ the ground set, $\Omega_{X}$ the choice domain, $A \in \Omega_{X}$ a menu, and elements of a menu items. For every menu $A \in \Omega_{X}, c(A)$ is the choice set of $A$, and comprises all items of $A$ that are deemed 'selectable' by the economic agent. If $c(A)$ is a singleton for any menu $A$, then we call $c$ a choice function, and identify it with the underlying map $c: \Omega_{X} \rightarrow X$ (defined in the obvious way). To simplify terminology, we often refer to a choice correspondence as a choice. The pair $(X, c)$ is a choice space.

Given a choice space $(X, c)$, a menu $A \in \Omega_{X}$ is improper if it is either a singleton or the whole ground set $X$; it is proper otherwise. Obviously all singletons are fixed points of $c$, that is, $c(\{x\})=\{x\}$ for each $x \in X$; thus, it suffices to define $c$ for menus with at least two items. Whenever dealing with finite menus, we shall simplify notation by underlining all selected items: thus, for instance, $\underline{a} \underline{b} d \underline{e} f$ stands for $c(\{a, b, d, e, f\})=\{a, b, e\}$.

The following notion of homomorphism between choice spaces plays a relevant role in our analysis, because most structural results given in this paper are stated 'up to isomorphisms'.

Definition 1 A homomorphism from a choice space $\left(X, c_{X}\right)$ to a choice space $\left(Y, c_{Y}\right)$ is a structure preserving map $\sigma: X \rightarrow Y$, that is, $\sigma\left(c_{X}(A)\right)=c_{Y}(\sigma(A))$ for each $A \in \Omega_{X}{ }^{4}{ }^{4}$ If $\sigma$ is a bijection, then it is an isomorphism; in this case, we say that $\left(X, c_{X}\right)$ and $\left(Y, c_{Y}\right)$ are isomorphic, and denote this fact by $\left(X, c_{X}\right) \cong\left(Y, c_{Y}\right)$.

Substructures of choice spaces are defined in the expected way:

Definition 2 Given a choice space $(X, c)$ and a nonempty set $X^{\prime} \subseteq X$, we define the subchoice (correspondence) $c \uparrow_{X^{\prime}}$ on $X^{\prime}$ by $c \uparrow_{X^{\prime}}(A):=c(A)$ for each $A \in \Omega_{X^{\prime}}=2^{X^{\prime}} \backslash\{\emptyset\}$. The pair $\left(X^{\prime}, c \uparrow_{X^{\prime}}\right)$ is called a choice subspace of $(X, c)$, denoted by $\left(X^{\prime}, c \uparrow_{X^{\prime}}\right) \sqsubseteq(X, c)$.

\subsection{One-point resolutions}

The core notion of this paper is that of one-point resolution.

Definition 3 Let $\left(X, c_{X}\right)$ and $\left(Y, c_{Y}\right)$ be disjoint choice spaces, i.e., $X \cap Y=\emptyset .{ }^{5}$ Select $x \in X$, and set $Z:=(X \backslash\{x\}) \cup Y$. The projection is the map $\pi: Z \rightarrow X$ defined by

$$
\pi(z):= \begin{cases}z & \text { if } z \in X \backslash\{x\} \\ x & \text { if } z \in Y .\end{cases}
$$

Then, the one-point resolution of $\left(X, c_{X}\right)$ at $x$ into $\left(Y, c_{Y}\right)$, denoted by

\footnotetext{
${ }^{4}$ As customary, we use the same notation for a map $\sigma: X \rightarrow Y$ and the (set-)map $\sigma: \Omega_{X} \rightarrow \Omega_{Y}$ induced by $\sigma$, defined by $A \mapsto \sigma(A)=\{\sigma(a): a \in A\}$ for all $A \in \Omega_{X}$.

${ }^{5}$ Disjointness of $X$ and $Y$ is a natural condition, which stems from the fact that the two choice spaces involved in a resolution must be mutually independent.
} 


$$
\left(Z, c_{Z}\right)=\left(X, c_{X}\right) \oplus_{x}\left(Y, c_{Y}\right)
$$

is the choice space $\left(Z, c_{Z}\right)$, where $c_{Z}: \Omega_{Z} \rightarrow \Omega_{Z}$ is the choice correspondence defined by

$$
c_{Z}(A):= \begin{cases}\left(c_{X}(\pi(A)) \backslash\{x\}\right) \cup c_{Y}(A \cap Y) & \text { if } x \in c_{X}(\pi(A)) \\ c_{X}(\pi(A)) & \text { otherwise }\end{cases}
$$

Then $\left(X, c_{X}\right)$ and $\left(Y, c_{Y}\right)$ are, respectively, the base (choice space) and the fiber (choice space), whereas the distinguished item $x \in X$ is the base point. The onepoint resolution $\left(X, c_{X}\right) \oplus_{x}\left(Y, c_{Y}\right)$ is said to be nontrivial if both $X$ and $Y$ contain at least two items, and trivial otherwise.

Notice that Definition 3 is sound, since the choice correspondence $c_{Z}$ is welldefined. Indeed, if $A \in \Omega_{Z}$ is such that $x \in c_{X}(\pi(A))$, then, in particular, $x \in \pi(A)$; it follows that $A \cap Y$ is nonempty, $c_{Y}(A \cap Y)$ makes sense, and $c_{Z}(A)$ is nonempty.

Remark 1 Trivial one-point resolutions are uninformative. Indeed, if $X=\{x\}$, then $Z=Y$, and so $\left(Z, c_{Z}\right)$ and $\left(Y, c_{Y}\right)$ are exactly the same. On the other hand, if $Y=\{y\}$, then $Z=(X \backslash\{x\}) \cup\{y\}$, and $\left(Z, c_{Z}\right)$ and $\left(X, c_{X}\right)$ are isomorphic.

For the sake of illustration, the next example describes a basic instance of resolution.

Example 4 (An elementary resolution) Let $\left(X, c_{X}\right)$ and $\left(Y, c_{Y}\right)$ be the choice spaces

$$
\left(X, c_{X}\right)=\{a, b\} \text { with } \underline{a} b \quad \text { and } \quad\left(Y, c_{Y}\right)=\{c, d\} \text { with } \underline{c} d .
$$

We determine $\left(Z, c_{Z}\right)=\left(X, c_{X}\right) \oplus_{a}\left(Y, c_{Y}\right)$. Clearly, $Z=\{b, c, d\}$. Next, we show that $c_{Z}$ is given by $b c d, b c, b d, c d$. Since the projection $\pi:\{b, c, d\} \rightarrow\{a, b\}$ is such that $\pi(b)=\bar{b}$ and $\pi(c)=\pi(d)=a$, we get $c_{X}(\pi(\{b, c, d\}))=c_{X}(\pi(\{b, c\}))=c_{X}(\pi(\{b, d\}))=c_{X}(\pi(\{c, d\}))=\{a\}$, and so the first line of definition (3) yields

$$
\begin{aligned}
& c_{Z}(\{b, c, d\})=\left(c_{X}(\pi(\{b, c, d\})) \backslash\{a\}\right) \cup c_{Y}(\{b, c, d\} \cap\{c, d\})=\emptyset \cup c_{Y}(\{c, d\})=\{c\}, \\
& c_{Z}(\{b, c\})=\left(c_{X}(\pi(\{b, c\})) \backslash\{a\}\right) \cup c_{Y}(\{b, c\} \cap\{c, d\})=\emptyset \cup c_{Y}(\{c\})=\{c\}, \\
& c_{Z}(\{b, d\})=\left(c_{X}(\pi(\{b, d\})) \backslash\{a\}\right) \cup c_{Y}(\{b, d\} \cap\{c, d\})=\emptyset \cup c_{Y}(\{d\})=\{d\}, \\
& c_{Z}(\{c, d\})=\left(c_{X}(\pi(\{c, d\})) \backslash\{a\}\right) \cup c_{Y}(\{c, d\} \cap\{c, d\})=\emptyset \cup c_{Y}(\{c, d\})=\{c\} .
\end{aligned}
$$

Hereafter, we shall be using a suggestive notation to identify one-point resolutions, e.g., in the case described above

$$
\underline{a} b \oplus_{a} \underline{c} d=b \underline{c} d, b \underline{c}, b \underline{d}, \underline{c} d .
$$

There are several possible interpretations of the notion of one-point resolution. Here is one in terms of a 'delegation of tasks', for two different settings. 
(Corporate structures) A company with a CEO and a single VP (of, say, the marketing department) makes choices over projects. The CEO decides: first, whether these decisions involve the marketing department or not; second, if marketing is involved, only then asking the VP to suggest choices; and, third, selecting one or more projects to undertake.

(Investment portfolios) An agent makes investments, possibly relying on a stock broker. The investor decides: first, whether to invest in stocks or not; second, if she decides to invest in stocks, only then asking the stock broker for recommendations; and, third, choosing one or more particular stocks to purchase.

Definition (3) is compact, and allows one to define special types of one-point resolutions on the basis of suitable properties of the projection (e.g., in a topological setting, requiring that the projection is continuous, or a quotient map, or closed, etc.).

The next result provides two alternative ways of looking at one-point resolutions. The first reformulation gives a taxonomy of cases based on the fact that the menu is either 'pure' (i.e., it is a subset of either $X$ or $Y$ ) or 'mixed' (i.e., it intersects both $X$ and $Y$ ). The second reformulation totally avoids using the projection, thus giving a more transparent—but unfortunately less agile—reading of the notion of one-point resolution.

Lemma 1 Let $\left(Z, c_{Z}\right)=\left(X, c_{X}\right) \oplus_{x}\left(Y, c_{Y}\right)$ be a one-point resolution. For any $A \in \Omega_{Z}$,

$$
c_{Z}(A)= \begin{cases}c_{X}(A) & \text { if } A \subseteq X \\ c_{Y}(A) & \text { if } A \subseteq Y \\ c_{X}(\pi(A)) & \text { if } A \cap X \neq \emptyset, A \cap Y \neq \emptyset, \text { and } x \notin c_{X}(\pi(A)) \\ \left(c_{X}(\pi(A)) \cup c_{Y}(A \cap Y)\right) \backslash\{x\} & \text { if } A \cap X \neq \emptyset, A \cap Y \neq \emptyset, \text { and } x \in c_{X}(\pi(A))\end{cases}
$$

$$
= \begin{cases}c_{X}(A) & \text { if } A \cap Y=\emptyset \\ c_{X}(A \cap X \cup\{x\}) & \text { if } A \cap Y \neq \emptyset \text { and } \cap \notin c_{X}(A \cap X \cup\{x\}) \\ \left(c_{X}(A \cap X \cup\{x\}) \cup c_{Y}(A \cap Y)\right) \backslash\{x\} & \text { if } A \cap Y \neq \emptyset \text { and } \cap \in c_{X}(A \cap X \cup\{x\}) .\end{cases}
$$

Lemma 1 decomposes the definition of one-point resolution into mutually exclusive cases. In (4), for a pure menu, both the base and the fiber are used to make the selection: see the first two lines of (4). Otherwise, for a mixed menu, look at whether the base point is chosen or not in the projection of the menu on $X$, and finally make the selection: see the last two lines of (4).

Formulation (5) yields a more direct interpretation. The agent partitions $Z$ into $X \backslash\{x\}$ and $Y$, where $x$ is some 'imaginary' alternative, and then she chooses from $A \in \Omega_{Z}$ as follows. If $A$ and $Y$ are disjoint (line 1), she only uses $c_{X}$. If $A$ and $Y$ intersect (lines 2 and 3), then she distinguishes whether the imaginary alternative $x$ is (i) not choosable from $A \cap X \cup\{x\}$, or (ii) choosable from it: in case (i), she uses $c_{X}$; in case (ii), she uses both $c_{X}$ and $c_{Y}$. In other words, the imaginary alternative $x$ captures the general attractiveness of the alternatives in $Y$, and, whenever $x$ is not desirable enough compared to alternatives in $A \cap X$, the agent ignores all alternatives in $Y$. 
Using substructures (see Definition 2), we can identify copies of the base and the fiber inside a one-point resolution:

Lemma $2 \operatorname{Let}\left(Z, c_{Z}\right)=\left(X, c_{X}\right) \oplus_{x}\left(Y, c_{Y}\right)$ be a one-point resolution. We have:

(i) $c_{Z} \uparrow_{Y}=c_{Y}$

(ii) $c_{Z} \uparrow_{X \backslash\{x\}}=c_{X} \uparrow_{X \backslash\{x\}}$;

(iii) for each $y \in Y, c_{Z} \uparrow_{X \backslash\{x\} \cup\{y\}} \cong c_{X}$ (via the projection $\pi$ ).

The following immediate consequence of Lemma 2 will be useful in Sect. 3: in fact, it will allow us to conclude that if a one-point resolution satisfies some properties of choice consistency, then so do both the base and the fiber.

Corollary 1 The base and the fiber of a one-point resolution are isomorphic to subspaces of the one-point resolution.

\subsection{Resolvable choices}

Not all choices can be seen as one-point resolutions of simpler choices. For instance, consider the following simple modification of Example 3.

Example 5 (Gemma's extended choice) Suppose Gemma's preferred restaurant also serves salmon $(m)$. Gemma likes salmon, but she prefers having it paired up with fish dishes (never with chips). In fact, her best choice is 'tuna \& salmon'. Accordingly, Gemma displays the following additional selections from the menus that include salmon:

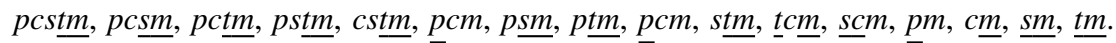

In the Appendix, we shall show that this choice cannot be seen as a nontrivial resolution.

In fact, the above choice is 'irresolvable' in the following sense:

Definition 4 A choice space $(Z, c)$ is resolvable if it is isomorphic to a nontrivial one-point resolution; otherwise, it is irresolvable. In what follows, we shall sometimes abuse notation, and call the choice $c$ resolvable whenever the choice space $(Z, c)$ is resolvable.

Resolvable choices can be arbitrarily complicated. On the other hand, the situation is quite simple on small ground sets, as the next example shows. 
Example 6 (Resolvability on small sets) On a 2-element set $X=\{a, b\}$, there are exactly two non-isomorphic choices, namely $\underline{a b}$ and $\underline{a b}$, and both are irresolvable. On a 3 -element set $X=\{b, c, d\}$, there are exactly five pairwise non-isomorphic resolvable choices, namely
(i) $\quad a \underline{b} \oplus_{a} \underline{c} d=\underline{b} c d, \underline{b} c, \underline{b} d, \underline{c} d$,
(ii) $\quad a \underline{b} \oplus_{a} \underline{c d}=\underline{b} c d, \underline{b} c, \underline{b} d, \underline{c d}$,
(iii) $\quad \underline{a} b \oplus_{a} \underline{c d}=\underline{b} \underline{c d}, \underline{b} \underline{c}, \underline{b} \underline{d}, \underline{c d}$,
(iv) $\quad \underline{a b} \oplus_{a} \underline{c d}=\underline{b c d}, \underline{b c}, \underline{b d}, \underline{c d}$,
(v) $\quad \underline{a b} \oplus_{a} \underline{c d}=\underline{b c} d, \underline{b c}, \underline{b d}, \underline{c d}$.

There are no other resolvable choices on $X$, since both the base and the fiber must be nontrivial. The one-point resolution (i) was explained in Example 4; the construction of other four resolved space is similar. In Sect. 5, we shall see that these five resolvable choices are also 'rationalizable', and there is one other choice on a 3-element set that is rationalizable but irresolvable.

For a ground set of size larger than three, finding whether a choice is resolvable or irresolvable can be more challenging. ${ }^{6}$

\subsection{Horizontal resolutions}

We conclude this preliminary part of the paper by showing that the notion of onepoint resolution is a special case of the following general ('horizontal') notion of resolution. $^{7}$

Definition 5 Let $\left(X, c_{X}\right)$ be a base choice space, and $\left\{\left(Y_{x}, c_{Y_{X}}\right)\right\}_{x \in X}$ a family of fiber choice spaces. Suppose all fiber sets $Y_{x}$ are pairwise disjoint, and they are also disjoint from the base set $X$. Set $Z:=\bigcup_{x \in X} Y_{x}$, and define the projection $\pi: Z \rightarrow X$ by $\pi(z):=x$ for all $x \in X$ and $z \in Y_{x}$. Let $c_{Z}: \Omega_{Z} \rightarrow \Omega_{Z}$ be the choice on $Z$ defined by

$$
c_{Z}(A):=\bigcup_{x \in c_{X}(\pi(A))} c_{x}\left(A \cap Y_{x}\right)
$$

for any $A$ in $\Omega_{Z}$. Notice that $c_{Z}$ is a well-defined choice, since $\emptyset \neq c_{Z}(A) \subseteq A$ for all nonempty $A \subseteq Z$. The choice space $\left(Z, c_{Z}\right)$ is called the horizontal resolution of $\left(X, c_{X}\right)$ into $\left(Y_{x}, c_{Y_{x}}\right)_{x \in X}$, and is denoted by $\left(X, c_{X}\right) \oplus\left(Y_{x}, c_{x}\right)_{x \in X}$.

\footnotetext{
${ }^{6}$ The descriptive characterization of resolvable choices given in this paper (Theorem 6 in Sect. 4), which is based on the notion of 'shrinkable menu', can be used for this, along with an effective algorithm for detecting shrinkability. Here by 'effective' we mean an algorithm whose complexity is polynomial in the majority of cases. Such an algorithm may employ the technique used in the proof of Example 5, given in the Appendix.

${ }^{7}$ A similar notion of horizontal resolution is introduced by Cantone et al. (2019) for some combinatorial structures, called 'convex geometries' by Edelman and Jamison (1985).
} 
It turns out that one-point resolutions and horizontal resolutions essentially have the same power in producing new choice spaces from given ones:

\section{Theorem 1}

(i) A one-point resolution of a base choice space $\left(X, c_{X}\right)$ is isomorphic to a horizontal resolution of $\left(X, c_{X}\right)$.

(ii) A horizontal resolution of a finite base choice space $\left(X, c_{X}\right)$ into $\left(Y_{x}, c_{Y_{x}}\right)_{x \in X}$ is isomorphic to a finite sequence of one-point resolutions at each of the points of $X$, in any order.

(iii) A horizontal resolution of an infinite base choice space $\left(X, c_{X}\right)$ into $\left(Y_{x}, c_{Y_{x}}\right)_{x \in X}$ is isomorphic to an inverse limit of one-point resolutions at the points of $X$.

The proof of part (i) of Theorem 1 is immediate. Indeed, given a onepoint resolution $\left(Z, c_{Z}\right)=\left(X, c_{X}\right) \oplus_{x_{0}}\left(Y, c_{Y}\right)$, take the family $\left(Y_{x}, c_{Y_{x}}\right)_{x \in X}$, where $Y_{x}:=Y$ and $c_{Y_{x}}:=c_{Y}$ if $x=x_{0}$, and $Y_{x}:=\{x\}$ otherwise. It is easy to check that $\left(Z, c_{Z}\right) \cong\left(X, c_{X}\right) \oplus\left(Y_{x}, c_{x}\right)_{x \in X}$.

On the contrary, the proof of parts (ii) and (iii) are rather long, although not conceptually difficult. The proof of (ii) is given in the Appendix. The proof of (iii) is more technical, and is available upon request.

Terminology. In view of Theorem 1, in this paper we only study one-point resolutions. Furthermore, we shall use the more agile term 'resolution' in place of 'onepoint resolution'.

\section{Consistency of resolutions}

We answer Question 1 from the Introduction, by showing that most of the properties of consistency considered in the literature are inherited (back and forth) by a resolution. We also prove that, on the contrary, WARP fails to be preserved in the forward direction, except in special cases, which we characterize.

\subsection{Rationalizable choices and axioms of choice consistency}

Here we present the basics of the theory of revealed preferences pioneered by Samuelson (1938), and successively developed (in chronological order) by Houthakker (1950), Chernoff (1954), Arrow (1959), Richter (1966), Sen (1971), and Herzberger (1973), among several others. See also the monograph by Chambers and Echenique (2016) for a recent perspective on revealed preference theory.

A weak preference on a nonempty set $X$ of alternatives is a reflexive relation $\succsim$ on $X$, where $x \gtrsim y$ means that $x$ is 'weakly preferred to' $y$. A weak preference $\gtrsim$ is the disjoint union of two derived relations: (1) the strict preference $>$, defined by 
$x>y$ if $x \gtrsim y$ and $\neg(y \gtrsim x)$; and (2) the indifference $\sim$, defined by $x \sim y$ if $x \gtrsim y$ and $y \gtrsim x$. A weak preference $\gtrsim$ on $X$ is complete (or total) if $x \gtrsim y$ or $y \gtrsim x$ holds for all distinct $x, y \in X$. Further, $\gtrsim$ is quasi-transitive if its strict preference $>$ is transitive, ${ }^{8}$ and acyclic if there are no $n \geq 3$ elements $x_{1}, x_{2}, \ldots, x_{n} \in X$ such that $x_{1}>x_{2}>\ldots>x_{n}>x_{1}$. Obviously, transitivity implies quasi-transitivity, and the latter property implies acyclicity. A weak preference is a preorder if it is transitive, a quasi-preorder if it is quasi-transitive, and a linear order if it is an antisymmetric total preorder. ${ }^{9}$

Given $\succsim$ on $X$, the set of maximal elements of a nonempty set $A \subseteq X$ is

$$
\max (A, \succsim)=\max (A,>):=\{a \in A: b>a \text { for no } b \in A\} .
$$

Notice that if $\gtrsim$ is complete, then the set of maximal elements of $A$ can be equivalently written as $\max (A, \succsim)=\{a \in A: a \succsim b$ for all $b \in A\}$. Observe also that, for any finite acyclic relation $\succsim$ on $X$, we have $\max (A, \succsim) \neq \emptyset$ for all nonempty $A \subseteq X$.

The rationality of a selection process is typically encoded by the possibility to justify it by maximizing a binary relation—see Samuelson (1938), Arrow (1959), and Sen (1971):

Definition 6 A choice $c: \Omega_{X} \rightarrow \Omega_{X}$ is rationalizable if there is a weak preference $\succsim$ on $X$ such that $c(A)=\max (A, \succsim)$ for all $A \in \Omega_{X}$. In particular, $c$ is:

- transitively rationalizable if it is rationalizable by a total preorder;

- quasi-transitively rationalizable if it is rationalizable by a preorder.

Clearly, a binary relation that rationalizes a (rationalizable) choice is acyclic. Further, for any rationalizable choice $c$, there are many weak preferences on $X$ that induce $c$. Indeed, what matters for the process of rationalization is only the asymmetric part of the rationalizing preference, whereas the derived indifference plays no role for this purpose. ${ }^{10}$ Thus, a choice $c$ is rationalizable if and only if there is a unique asymmetric relation that generates $c$ by maximizing over menus.

It is well-known that the rationalizability of a choice is connected to the satisfaction of suitable axioms of choice consistency, which are formulas codifying rules of coherent behavior of an economic agent. Among the several properties of this kind that are considered in the literature, the following are relevant to our analysis:

$\checkmark$ Axiom $(\alpha)$ (standard contraction consistency): for any $A, B \in \Omega_{X}$ and $x \in A$, if $A \subseteq B$ and $x \in c(B)$, then $x \in c(A)$.

\footnotetext{
${ }^{8}$ Recall that a (binary) relation $R$ on $X$ is transitive if $x R y R z$ implies $x R z$ for all $x, y, z \in X$.

${ }^{9}$ Recall that a relation $R$ on $X$ is antisymmetric if $(x R y) \wedge(y R x)$ implies $x=y$ for all $x, y \in X$.

${ }^{10}$ However, indifference becomes relevant to identify indecisive behavior: see Eliaz and Ok (2006) for the notion of a regular preorder, and Giarlotta and Watson (2017) for some notions of revealed similarity.
} 
$\diamond$ Axiom $(\gamma)$ (standard expansion consistency): for any $\left\{A_{i}: i \in I\right\} \subseteq \Omega_{X}$ and $x \in \bigcap_{i \in I} A_{i}$, if $x \in c\left(A_{i}\right)$ for all $i \in I$, then $x \in c\left(\bigcup_{i \in I} A_{i}\right)$.

$\diamond \operatorname{Axiom}(\beta)$ (symmetric expansion consistency): for any $A, B \in \Omega_{X}$ and $x, y \in A$, if $A \subseteq B, x, y \in c(A)$, and $y \in c(B)$, then $x \in c(B)$.

$\checkmark$ Axiom ( $\rho$ ) (standard replacement consistency): for any $A \in \Omega_{X}, y \in A$, and $x \in X \backslash A$, if $y \in c(A)$ and $y \notin c(A \cup\{x\})$, then $x \in c(A \cup\{x\})$.

WARP (Weak Axiom of Revealed Preference): for any $A \in \Omega_{X}$ and $x \in A$, if there are $y \in c(A)$ and $B \in \Omega_{X}$ such that $y \in B$ and $x \in c(B)$, then $x \in c(A)$.

$\checkmark$ WARNI (Weak Axiom of Revealed Non-Inferiority): for any $A \in \Omega_{X}$ and $x \in A$, if for each $y \in c(A)$ there is $B \in \Omega_{X}$ with $x \in c(B)$ and $y \in B$, then $x \in c(A)$.

Axiom $(\alpha)$ is studied by Chernoff (1954), whereas axioms $(\gamma)$ and $(\beta)$ are due to Sen (1971). Axiom ( $\rho$ ) is very recent (Cantone et al. 2016). WARP is introduced in Samuelson (1938), and WARNI is the weakening of WARP due to Eliaz and Ok (2006).

The interpretation of these properties is natural. Chernoff's axiom $(\alpha)$ says that if an item is selected from a menu $B$, then it is still selected from any submenu $A \subseteq B$ containing it. Sen's axiom $(\gamma)$ states that if an item is selected from all menus in a family $\mathcal{F}$, then it is also selected from the menu obtained as the union of the elements of $\mathcal{F}$. Sen's axiom $(\beta)$ says that if two items are selected from a menu $A$, then they are simultaneously either selected or rejected in any larger menu $B$. Axiom $(\rho)$ states that if an item $y$ is selected from a menu $A$ but not from the larger menu $A \cup\{x\}$, then the added item $x$ is selected from $A \cup\{x\}$. WARP summarizes features of contraction and expansion consistency in a single axiom: it says that an item $x$ is selected from a menu $A$ whenever there is an item $y$ selected from $A$ with the property that $x$ is chosen from some other menu $B$ containing both $x$ and $y$. WARNI is a weaker version of WARP.

The next result collects some known relations between the rationalizability of a choice correspondence and all axioms of choice consistency introduced so far. ${ }^{11}$ On this point, see Arrow (1959), Sen (1971), and Cantone et al. (2016). ${ }^{12}$

Theorem (Rationalization by Axioms) For any choice c, we have:

(i) $c$ is rationalizable $\Longleftrightarrow(\alpha)$ and $(\gamma)$ hold.

(ii) $c$ is transitively rationalizable $\Longleftrightarrow W A R P$ holds $\Longleftrightarrow(\alpha)$ and $(\beta)$ hold.

(iii) $c$ is quasi-transitively rationalizable $\Longleftrightarrow W A R N I$ holds $\Longleftrightarrow(\alpha)$, $(\gamma)$, and $(\rho)$ hold.

\footnotetext{
11 Some of the stated results hold for choices defined on more general domains. However, in the general case, WARNI is stronger than quasi-transitive rationalizability, whereas the joint satisfaction of axioms $(\alpha),(\gamma)$, and $(\rho)$ is equivalent to the latter property: see Cantone et al. (2016).

12 In particular, Cantone et al. (2016) provide a taxonomy of rationalizable choices, which are classified according to the transitive degree of the rationalizing preference. These 'degrees of transitivity', called $(m, n)$-Ferrers properties (Giarlotta and Watson 2014,2018$)$, give rise to new axioms $\left(\rho_{m, n}\right)$ of choice consistency, which are variations of standard replacement $(\rho)$.
} 


\subsection{Backward preservation of axioms of choice consistency}

The process to show that a property $\mathcal{P}$ is preserved by resolutions consists of two steps:

(Backward Preservation) if $\left(X, c_{X}\right) \oplus_{x}\left(Y, c_{Y}\right)$ satisfies $\mathcal{P}$, then $\left(X, c_{X}\right)$ and $\left(Y, c_{Y}\right)$ satisfy $\mathcal{P}$;

(Forward Preservation) if $\left(X, c_{X}\right)$ and $\left(Y, c_{Y}\right)$ satisfy $\mathcal{P}$, then $\left(X, c_{X}\right) \oplus_{x}\left(Y, c_{Y}\right)$ satisfies $\mathcal{P}$.

Backward preservation is easy to prove, because it follows from a general result about the preservation of suitable formulas by passing to subspaces. Indeed, upon calling a property $\mathcal{P}$ of choice spaces hereditary whenever its satisfaction by any choice space $(X, c)$ implies its satisfaction by any subspace of $(X, c)$, Corollary 1 readily yields

Lemma 3 If a resolution satisfies a hereditary property, then so do its base and its fiber.

Observe that every property $\mathcal{P}$ of choice correspondences that can be expressed in a 'purely universally quantified' manner by a formula $\Phi_{\mathcal{P}}$ is hereditary, provided that the domain is closed with respect to the operatations involved in $\Phi_{\mathcal{P}}{ }^{13}$ This is the case for all basic axioms of consistency examined in this paper. ${ }^{14}$ Thus Lemma 3 readily yields their backward preservation. On the contrary, forward preservation is harder to prove (and fails in some cases).

\subsection{Preservation of rationalizability and quasi-transitivity}

The operation of resolution preserves the basic model of rational choice. To wit, here we show that properties $(\alpha),(\gamma)$, and $(\rho)$ are preserved back and forth by resolutions. Since properties $(\alpha),(\gamma)$, and $(\rho)$ are hereditary, backward preservation follows from Lemma 3. On the other hand, a proof by cases yields forward preservation. Summing up, we have:

Theorem 2 A resolution satisfies property $(\alpha)$ (respectively, $(\gamma),(\rho)$ ) if and only its base and its fiber satisfy $(\alpha)$ (respectively, $(\gamma),(\rho))$.

${ }^{13}$ By a 'purely universally quantified formula' we mean any formula of the following form

$$
\left(\forall \mathcal{F}_{1}\right) \ldots\left(\forall \mathcal{F}_{m}\right)\left(\forall X_{1}\right) \ldots\left(\forall X_{n}\right)\left(\forall x_{1}\right) \ldots\left(\forall x_{k}\right) \varphi
$$

in which $\mathcal{F}_{1}, \ldots, \mathcal{F}_{m}$ range over collections of menus, $X_{1}, \ldots, X_{n}$ range over menus, $x_{1}, \ldots, x_{k}$ range over items, and $\varphi$ is a quantifier-free formula that involves the standard set operations and constants.

${ }^{14}$ Notice that the formulation of WARP we have chosen is not purely universally quantified. However, there are many equivalent formulations of WARP, and some of them are of the claimed type. 
From Theorem 2 and Theorem 'Rationalization by Axioms', it readily follows

Corollary 2 A resolution is rationalizable if and only if both its base and its fiber are rationalizable. A resolution is quasi-transitively rationalizable if and only if both its base and its fiber are quasi-transitively rationalizable.

Corollary 2 can be suggestively rephrased as 'rationality factors through resolutions'. For instance, in a corporate structure setting, if the CEO makes a rationalizable selection and delegates all marketing choices to a VP whose selections are rationalizable, then the overall selection is still rationalizable.

\subsection{Preservation of path independence}

Resolutions also preserve (back and forth) some additional axioms of choice consistency. Here we show that the following well-known property, usually attributed to Plott (1973), belongs to this category of axioms:

$\diamond \mathbf{P I}\left(\right.$ Path Independence): for any $A, B \in \Omega_{X}, c(A \cup B)=c(c(A) \cup c(B))$.

As Plott (1973) puts it, path independence stems for the fact that a dynamic process of selection often proceeds in a 'divide and conquer' manner: options are split up into smaller sets, a choice is made over each of these sets, the selected items are collected, and then a choice is made from them. Path independence means that the final outcome is independent of the way the alternatives are initially divided up for consideration. $^{15}$

For finite choices, PI can be decomposed into $(\alpha)$ and $(\rho)$ : see, e.g., Aizerman and Malishevski (1981) and Moulin (1985). However, this decomposition does not hold in the infinite case, since there are choices satisfying $(\alpha)$ and $(\rho)$ for which PI fails. ${ }^{16}$ Thus, to show that $\mathrm{PI}$ is preserved by resolutions, we need a direct argument, which yields

Theorem 3 A resolution is path independent if and only if both its base and its fiber are path independent.

\footnotetext{
15 The original explanation given by Plott (1973) works well for disjoint menus $A$ and $B$. However, since $A$ and $B$ may intersect, the notion of path independence also covers some additional cases.

16 To prove the claim, let $c: 2^{X} \rightarrow 2^{X}$ be the choice on $X:=\mathbb{N} \cup\left\{x_{0}\right\}$ defined by
}

$$
c(A):= \begin{cases}\{\min A\} & \text { if } x_{0} \notin A \\ \left\{\min (A \cap \mathbb{N}), x_{0}\right\} & \text { if } x_{0} \in A \text { and } 2 \leq|A|<\infty \\ \left\{x_{0}\right\} & \text { if } x_{0} \in A \text { and }(|A|=\infty \text { or }|A|=1) .\end{cases}
$$

Notice that $c$ fails to be rationalizable, since $(\gamma)$ does not hold: indeed, $0 \in c(\mathbb{N}) \cap c\left(\left\{0, x_{0}\right\}\right)$, however $0 \notin c\left(\mathbb{N} \cup\left\{0, x_{0}\right\}\right)=c(X)$. The reader may easily verify that $c$ satisfies both $(\alpha)$ and $(\rho)$, but not $\mathrm{PI}$. 


\subsection{Preservation of rationalizability by two sequential criteria}

In their model of bounded rationality, Manzini and Mariotti (2007) present an approach to explain choice behavior, which employs a finite number of binary preferences (called 'rationales') in a fixed order for all available menus. In particular, a choice $c$ is 2-sequentially rationalizable if there is an ordered pair $\left(>_{1},>_{2}\right)$ of asymmetric relations on $X$ such that $c(A)=\max \left(\max \left(A,>_{1}\right),>_{2}\right)$ for all $A \in \Omega_{X}{ }^{17}$

Manzini and Mariotti (2007) characterize the 2-sequential rationalizability of choice functions by the satisfaction of standard expansion $(\gamma)$ and a weaker form of WARP, which is stated as follows for choice correspondences:

WWARP (Weak WARP): for any $A, B \in \Omega_{X}$ and $x, y \in A$, if $A \subseteq B$, $y \notin c(\{x, y\})$, and $x \in c(B)$, then $y \notin c(A)$.

The characterization of 2-sequential rationalizability proved by Manzini and Mariotti (2007) for choice functions does not hold for choice correspondences, as GarcíaSanz and Alcantud (2015) show. However, under a weak property of choice consistency, namely

CWDE (Choosing Without Dominated Elements): for any $A \in \Omega_{X}$ and $x, y \in A$, if $y$ never chosen in menus containing $x$ and $y$, then $c(A)=c(A \backslash\{y\})$,

Manzini and Mariotti's characterization still holds. In fact, we have:

Lemma 4 (García-Sanz and Alcantud 2015) A choice correspondence satisfying CWDE is 2-sequentially rationalizable if and only if properties $(\gamma)$ and WWARP hold for it.

Our ideal goal is to show that 2-sequential rationalizability is preserved by resolutions. To that end, we would need a characterization of 2-sequential rationalizability, which, to the best of our knowledge, is not available yet. Thus, here we only prove a partial result: ${ }^{18}$

Theorem 4 Let $\left(Z, c_{Z}\right)=\left(X, c_{X}\right) \oplus_{x}\left(Y, c_{Y}\right)$ be a resolution such that $\left(Z, c_{Z}\right),\left(X, c_{X}\right)$, and $\left(Y, c_{Y}\right)$ satisfy CWDE. Then $\left(Z, c_{Z}\right)$ is 2-sequentially rationalizable if and only if both $\left(X, c_{X}\right)$ and $\left(Y, c_{Y}\right)$ are 2-sequentially rationalizable.

\footnotetext{
${ }^{17}$ Manzini and Mariotti (2007) define this notion for a choice function (i.e., single-valued). The extension to a choice correspondence (i.e., possibly multi-valued) is due to García-Sanz and Alcantud (2015).

${ }^{18}$ Notice that any attempt to obtain a better result for resolutions on the basis of Lemma 4 fails, because CWDE is not hereditary.
} 


\subsection{Limited preservation of WARP}

Since WARP is a hereditary property, Lemma 3 ensures that it is backward preserved by resolutions. On the contrary, forward preservation does not hold, in general.

Example 7 Consider the following resolution: $\underline{a b} \oplus_{a} \underline{c} d=\underline{b c} d, \underline{b c}, \underline{b d}, \underline{c} d$. Both the base and the fiber satisfy WARP, whereas the resolved choice does not, because property $(\beta)$ fails: indeed, both items in $\{b, d\}$ are selected, but $b$ is selected from the larger menu $\{b, c, d\}$ whereas $d$ is not. (Notice that, according to Corollary 2, the resolved choice is quasi-transitively rationalizable, since it satisfies properties $(\alpha),(\gamma)$, and $(\rho)$.)

Here we characterize all cases in which WARP is preserved forward. As we shall see, forward preservation depends on either the features of the base point (which must be very special) or those of the fiber choice (which must be trivial).

Definition 7 Given a choice $(X, c)$, an item $x \in X$ is called a repellent point for $(X, c)$ if, for all menus $A \in \Omega_{X}$, either $c(A)=\{x\}$ or $x \notin c(A)$ holds. Equivalently, $x$ is repellent if $x \in c(A)$ implies $c(A)=\{x\}$ for each $A \in \Omega_{X}$.

Notice that, for a choice satisfying WARP, a repellent point must always be either at the top or at the bottom of the total preorder that rationalizes the choice. Then, we have:

Theorem 5 The following statements are equivalent for $\left(Z, c_{Z}\right)=\left(X, c_{X}\right) \oplus_{x}\left(Y, c_{Y}\right)$ :

(i) $\left(Z, c_{Z}\right)$ satisfies axiom $(\beta)$;

(ii) $\left(X, c_{X}\right)$ and $\left(Y, c_{Y}\right)$ satisfy axiom $(\beta)$, and either $x$ is a repellent point of $\left(X, c_{X}\right)$ or $c_{Y}$ is the identity map (or both).

Theorems 2 and 5, along with Theorem 'Rationalization by Axioms', readily yield

Corollary 3 A resolution satisfies WARP if and only its base and its fiber satisfy $W A R P$, and either the base point is repellent or the choice restricted to the fiber choice is the identity. Equivalently, a resolution is transitively rationalizable if and only if so are its base and its fiber, and either the base point is repellent or the fiber choice is the identity.

\section{Characterizing resolvability}

Here we constructively answer Question 2 in the Introduction by identifying special menus. 


\subsection{Shrinkable menus}

Definition 8 A menu $E \in \Omega_{Z}$ in a choice space $(Z, c)$ is shrinkable if, for any $A \in \Omega_{Z}$,

(S1) if $A \cap E \neq \emptyset$, then $c(A) \backslash E=c(A \cup E) \backslash E$;

(S2) if $c(A) \cap E \neq \emptyset$, then $c(A) \cap E=c(A \cap E)$;

(S3) if $A \cap E \neq \emptyset$, then $c(A \cup E) \cap E \neq \emptyset$ if and only if $c(A) \cap E \neq \emptyset$.

Notice that improper menus (i.e., singletons and the whole ground set) are clearly shrinkable. The conditions of shrinkability (S1)-(S3) can be interpreted in the two settings described in Section 2.1.

(Corporate structures) In a corporation with a CEO and a VP of marketing:

(S1) the non-marketing tasks chosen by the CEO do not depend on which marketing tasks are available, as long as at least one is;

(S2) the tasks chosen by the VP do not depend on what non-marketing tasks are available;

(S3) whether or not a marketing task is chosen is unaffected by which marketing tasks are available, as long as at least one is.

(Investment porftfolios) In an investment portfolio setting with a trusted stock broker:

- (S1) the non-stock investments purchased do not depend on which stocks are available, as long as at least one is;

- (S2) the particular stocks purchased by the investor do not depend on what non-stock investments are available;

- (S3) whether or not the investor purchases stocks at all is unaffected by which good stocks are available, as long as at least one is.

In these two settings, a shrinkable menu reveals (i) an autonomous department within the corporation, or (ii) an implicit trusted stock broker on whom the investor relies.

\subsection{Main result}

We aim at characterizing resolvable choices. To start, we describe how a choice space with a shrinkable menu canonically induces another choice space.

Definition 9 Let $(Z, c)$ be a choice space, and $Y \in \Omega_{Z}$ a shrinkable menu. Select $x \notin Z$, set $X:=(Z \backslash Y) \cup\{x\}$, and let $\pi: Z \rightarrow X$ be the projection, defined by $\pi(z):=z$ if $z \in Z \backslash Y$ and $\pi(z):=x$ if $z \in Y$. Define a choice $c_{X}: \Omega_{X} \rightarrow \Omega_{X}$ on $X$ by setting, for any $A \in \Omega_{X}$, 


$$
c_{X}(A):= \begin{cases}c(A) & \text { if } x \notin A \\ \pi(c((A \backslash\{x\}) \cup Y)) & \text { if } x \in A .\end{cases}
$$

We call $\left(X, c_{X}\right)$ the base choice space induced by $(Z, c)$ and $Y$ (relative to $x$ ).

The idea underlying the definition of induced choice space is the following. The input is a primitive choice space $(Z, c)$, of which we wish to understand whether it is resolvable or not. To that end, we enquire about the existence of a shrinkable menu $Y$ in $(Z, c)$, which inherits a choice structure $c \uparrow_{Y}$ from the original space. If such a shrinkable menu $Y \in \Omega_{Z}$ exists, then we already have two of the three choice spaces involved in a resolution: the resolved space $(Z, c)$ and the fiber space $\left(Y, c \uparrow_{Y}\right)$. We need a canonical way to define the base space $\left(X, c_{X}\right)$ and the base point $x$ : Definition 9 does the job in a natural way.

Specifically, first we create the ground set $X$ of $\left(X, c_{X}\right)$ in the only possible way: take any point $x$ outside $Z$, and join it to the set of all points of $Z$ that do not belong to $Y$. Second, we define the choice correspondence $c_{X}$ by using the primitive choice $c$ and the projection $\pi$ as follows: for each menu $A \in 2^{X}$ that is a subset of $Z$, we set $c_{X}:=c$; on the other hand, if $A \in 2^{X}$ contains $x$, then first we apply $c$ to the menu $A^{\prime} \in 2^{Z} \backslash 2^{X}$ obtained from $A$ by substituting $x$ by $Y$, and successively project this choice set onto $X$.

We are left to prove that this process really yields a decomposition of the primitive choice in all cases in which there is a shrinkable menu. The next result witnesses this fact.

Theorem 6 Let $(Z, c)$ be a choice, and $Y \in 2^{Z}$ a shrinkable menu. If $\left(X, c_{X}\right)$ is the base choice induced by $(Z, c)$ and $Y$ (relative to some $x \notin Z$ ), then $(Z, c)=\left(X, c_{X}\right) \oplus_{x}\left(Y, c \uparrow_{Y}\right)$.

The proof of Theorem 6 is based on the following technical result:

Lemma 5 Let $(Z, c)$ be a choice, and $E \in 2^{Z}$ a shrinkable menu. For each $A \in 2^{Z}$ such that $A \cap E \neq \emptyset$, we have:

$$
c(A)= \begin{cases}(c(A \cup E) \backslash E) \cup c(A \cap E) & \text { if } c(A \cup E) \cap E \neq \emptyset \\ c(A \cup E) & \text { if } c(A \cup E) \cap E=\emptyset .\end{cases}
$$

The proof of Lemma 5 is easy, and is left to the reader. However, it is useful to describe how properties (S1)-(S3) are used to determine the choice set $c(A)$ of a menu $A$ that intersects a shrinkable menu $E$. Specifically, properties (S1) and (S3) are needed to prove the equality in the second line, that is, $c(A)=c(A \cup E)$ whenever $E$ does not intersect $c(A \cup E)$. On the other hand, property (S2) yields the first equality, that is, $c(A)=(c(A \cup E) \backslash E) \cup c(A \cap E)$ whenever $E$ intersects $c(A \cup E)$. 
From Theorem 6, we readily derive a characterization of resolvability:

\section{Corollary 4 A choice is resolvable if and only if there is a shrinkable proper menu.}

To efficiently use the characterization in Corollary 4, we need a fast algorithm that can detect the existence of a shrinkable menu when given data describing choice behavior. We have some partial results in this direction, which are related to the proof of Example 5 in the Appendix, where we suggest that the computational complexity of detecting resolvability is exponential, but nevertheless provide an algorithm that runs in polynomial (cubic) time in most of the practical cases.

We can now complete Example 3 of the Introduction, and formally write Gemma's choice at her preferred restaurant as a resolution of smaller choices.

Example 8 (Resolvability of Gemma's choice) Recall from Example 3 the formal definition of Gemma's choice behavior at the restaurant: pcst, pcs, pct, pst, cst, pc, $p s, p t, \underline{c s}, \underline{c t}, \underline{s t}$. It is easy to show that the proper menu $\{s, t\}=\{\overline{\mathrm{sea}} \overline{\mathrm{b}}$ ass, tuna $\}$, in which sea bass is preferred over tuna, is shrinkable. Thus, by Corollary 4, Gemma's choice is resolvable. Next, we use Theorem 6 to explicitly exhibit a decomposition of her choice behavior in a base and a fiber. First we compute the base choice on $\{p, c, f\}=\{$ pizza, chips, fish $\}$ induced by $\{s, t\}$, obtaining $p \underline{f}, p c, p f, \underline{f}$. This shows that Gemma selects fish and chips if both are available, and selects pizza alone over fish or chips if only one of the latter is available. (Notice that Gemma's selection between pizza and fish-and-chips does not depend on whether either sea bass or tuna or both are available: this is the independence condition at the basis of the decomposition process.) Now Theorem 6 yields an explicit resolution of Gem-

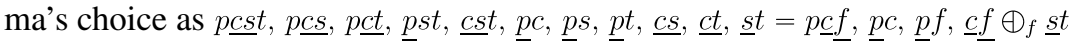

\subsection{Shrinkability and indiscernibility}

In this section we briefly discuss a notion that is related to shrinkability, which is called indiscernibility. ${ }^{19}$ In fact, we shall explain why an alternative name for 'shrinkable menu' may be 'outer indiscernible menu'. To start, we recall the notion of an indiscernible menu from Cantone et al. (2019): ${ }^{20}$

Definition 10 Let $(Z, c)$ be a choice space. A menu $E \in \Omega_{Z}$ is indiscernible if the following two properties hold for each $A \in \Omega_{Z}$ :

\footnotetext{
19 The indiscernibility of a menu encodes Leibniz's principle of the 'identity of indiscernibles' within choice theory. On this principle, see Leibniz (1966).

${ }^{20}$ To be precise, the notion of an indiscernible menu is originally defined for a finite choice space, and moreover in a different way: see Definition 9 in Section 4.1 of Cantone et al. (2019). The definition given here is the characterization of an indiscernible menus proved in Appendix A of the mentioned paper, where instead no finiteness is assumed.
} 
(I1) if $A \cap E \neq \emptyset$, then $c(A)=c(A \cup E) \cap A$;

(I2) if $c(A) \cap E \neq \emptyset$, then $c(A) \cap E=A \cap E$.

The indiscernibility of a menu $E$ intuitively says that the choice distinguishes neither the external relationships of $E$ with other menus nor the internal structure of $E$. The next result links indiscernibility and shrinkability (hence, by Theorem 6, resolvability).

Theorem 7 The following statements are equivalent for a menu E:

(i) E is indiscernible;

(ii) $E$ is shrinkable and $c \uparrow_{E}$ is the identity $\mathrm{id}_{2^{E}}$.

In particular, a choice space in which there is an indiscernible proper menu is resolvable.

The condition $c \uparrow_{E}=\mathrm{id}_{2^{E}}$ essentially says that the menu $E$ is 'inner indiscernible'. Thus, Theorem 7 can be restated as follows: A menu is indiscernible if and only if it is outer and inner indiscernible. Below we interpret Theorem 7 in the usual two settings.

(Corporate structures) If a department is 'mindless' (every possible task is chosen), then those choices are indistinguishable by the corporation. If certain actions always go together, then they can be viewed as the actions of a mindless department.

(Investment portfolios) If an investor gives her stock broker carte blanche (all available good stocks are chosen), then the investor does not distinguish between good stock investments. If an investor always chooses certain stocks together, when available, then these stocks can be viewed as chosen by a stock broker with carte blanche.

We close this section with a remark that sheds light on the notion of indiscernible menu.

Remark 2 Define on a choice space $(X, c)$ a binary relation of revealed indiscernibility as follows: two items $x, y \in X$ are (revealed to be) indiscernible if the menu $\{x, y\}$ is indiscernible. Revealed indiscernibility is an equivalence relation on $X$, which preserves the choice structure and yields a fully informative quotient choice space. More precisely, whenever the ground set $X$ is finite, revealed indiscernibility is the maximum 'congruence relation' on $(X, c)$ : see Cantone et al. (2019) for details. ${ }^{21}$

\footnotetext{
21 See also Giarlotta and Watson (2017), where revealed indiscernibility is paired up with other symmetric binary relations to generate what is called a 'necessary and possible indifference'.
} 


\section{Rationalizability and resolvability}

We (partially) answer Question 3 of the Introduction, examining the relationship between rationalizability and resolvability. There is a remarkable intersection between these notions, which provides a further motivation for our analysis in the spirit of revealed preference theory: all nontrivial choices that are rationalizable by a total preorder (i.e., satisfying WARP) are resolvable. ${ }^{22}$ Figure 2 summarizes the main findings of this section.

The simple idea developed in this section is that rationalizability makes resolvability a problem about directed graphs (or, equivalently, binary relations) rather than choice. That is, when choice is rationalizable, resolvability can be rewritten as a requirement on the "rationalizing" directed graph.

The next example compares rationalizability and resolvability for choices on a small ground set.

Example 9 (Rationalizable vs resolvable on a 3-element set) Let $X=\{b, c, d\}$. There are exactly six pairwise non-isomorphic rationalizable choices on $X$. Five of them are the resolvable choices (i)-(v) described in Example 6. In fact, choices (i)-(iv) are rationalizable by a transitive revealed preference, whereas choice (v) satisfies properties $(\alpha),(\gamma)$ and $(\rho)$, but not WARP. The sixth rationalizable (but irresolvable) choice on a 3-element set is

$$
\text { (vi) } \underline{b} c d, \underline{b c}, \underline{b d}, \underline{c d} \text {. }
$$

Notice that this last choice fails to satisfy property $(\rho)$ : indeed, $d$ is chosen in $A=\{b, d\}$ and not in $A \cup\{c\}=Z$, but $c$ is not selected from $Z$.

\subsection{Resolvability generalizes WARP}

The main result of this section is the following:

Theorem 8 For any choice space with at least three items, WARP implies resolvability.

Given the relevance of Theorem 8, below we sketch the argument used to prove it.

1. Let $\left(Z, c_{Z}\right)$ be a choice space satisfying WARP. By Theorem 'Rationalization by Axioms' part (ii), the choice correspondence $c_{Z}$ is rationalizable by a total preorder $\gtrsim_{Z}$. Two cases: (a) $\gtrsim_{Z}$ is a linear order; (b) $\gtrsim_{Z}$ is a total preorder that fails to be a linear order.

\footnotetext{
${ }^{22}$ As a matter of fact, more is true. Indeed, additional types of rationalizable choices, which are justified by special types of preorders, are resolvable as well: see Cantone et al. (2020a).
} 


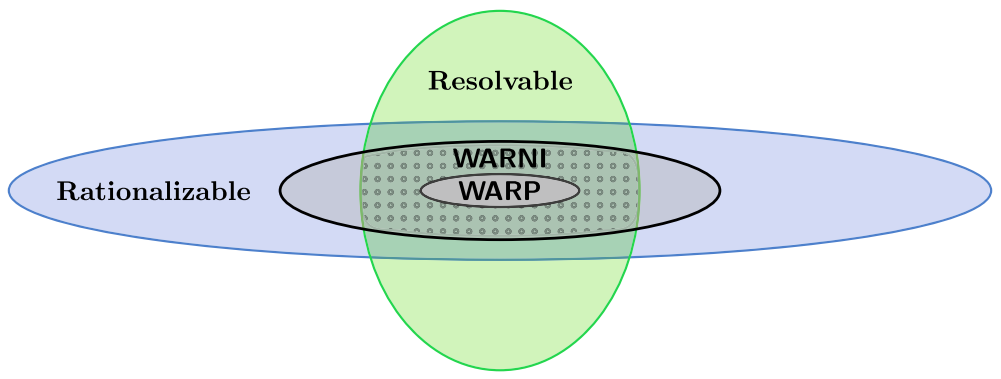

Fig. 2 Independence and connection between rationalizability and resolvability. The family of choices (on a ground set with at least three items) satisfying WARP is contained in the intersection of the families of rationalizable choices and resolvable choices (Theorem 8). A similar inclusion does not hold for the family of choices rationalizable by a preorder, i.e., satisfying WARNI (cf.: Examples 11 and 12). Furthermore, the dotted area is nonempty (Example 13)

2. In case (a), there exists distinct $z_{1}, z_{2} \in Z$ that are consecutive with respect to $>_{Z}$ (i.e., $z_{1}>_{Z} z_{2}$ holds, and there is no $z \in Z$ such that $z_{1}>_{Z} z>_{Z} z_{2}$ ). Then the menu $\left\{z_{1}, z_{2}\right\}$ is shrinkable, hence $\left(Z, c_{Z}\right)$ is resolvable by Corollary 4 .

3. In case (b), there exists distinct $z_{1}, z_{2} \in Z$ that are indifferent with respect to $\gtrsim_{Z}$. Then the menu $\left\{z_{1}, z_{2}\right\}$ is indiscernible, hence $\left(Z, c_{Z}\right)$ is resolvable by Theorem 7 .

The above proof shows some leverage to extend Theorem 8 to other rationalizable choices. In fact, at step 3, the resulting menu is indiscernible, which, by Theorem 7, is stronger than saying that it is shrinkable. It turns out that Theorem 8 can be generalized to the case of choices that are rationalizable by a certain type of preorder (which encompasses a total preorder as a special case). Since the proof of the latter fact requires a long detour into the process of decomposition of preferences, we shall deal with this topic in a subsequent paper (Cantone et al. 2020a).

The next example exhibits a minimal instance of the fact that resolvability does not imply rationalizability; in particular, the converse of Theorem 8 is false.

Example 10 (Resolvable and non-rationalizable) Resolve any non-rationalizable choice on a 3-element set at a point into a 2-element choice space such that its two items are indifferent. The resulting choice space is resolvable (by construction) but not rationalizable (since otherwise, by Corollary 2 , the base choice would be rationalizable as well).

\subsection{Quasi-transitive rationalizability vs resolvability}

A natural question is whether Theorem 8 can be extended to all choices that are rationalizable by an incomplete preorder. The answer is negative: 
Proposition 1 Quasi-transitive rationalizability and resolvability are independent.

To justify Proposition 1, below we exhibit an irresolvable choice that is rationalizable by a preorder (Example 11), and a resolvable choice that is rationalizable by a preference with an intransitive strict part (Example 12).

Example 11 (WARNI and irresolvable) Define a weak preference $\gtrsim$ on $X=\{x, y, z, t\}$ by letting $x \sim y, x>z, x>t, y \sim z, y>t$, and $z \sim t$. It is easy to check that $\gtrsim$ is quasi-transitive and total, but not transitive. ${ }^{23}$ Let $c_{\gtrsim}: \Omega_{X} \rightarrow \Omega_{X}$ be the choice correspondence derived from $\succsim$, which is defined by $c_{\gtrsim}(A):=\max (A, \succsim)$ for all $A \in \Omega_{X}$. By construction, the choice space $\left(X, c_{\gtrsim}\right)$ is quasi-transitively rationalizable, hence WARNI-equivalently, properties $(\alpha),(\gamma)$, and $(\rho)$-holds by Theorem 'Rationalization by Axioms' part (iii). However, $\left(X, c_{\gtrsim}\right)$ is irresolvable by Corollary 4 , because there is no proper shrinkable menu. (The long but straightforward verification of the last fact is left to the reader.)

Example 12 (Resolvable and rationalizable, but not WARNI) Define a weak preference $\succsim$ on $X=\{x, y, z\}$ by letting $x>y, x \sim z$, and $y>z$. It is easy to check that $\gtrsim$ is acyclic and total, but not quasi-transitive. Let $c_{\gtrsim}: \Omega_{X} \rightarrow \Omega_{X}$ be the choice correspondence derived from $\gtrsim$, that is, $x y, x z, y z$, and $x y z$. By construction, the choice space $\left(X, c_{\gtrsim}\right)$ is rationalizable but not quasi-transitively rationalizable, hence WARNI fails by Theorem 'Rationalization by Axioms' part (iii) ${ }^{24}$ Let $\left(Y, c_{Y}\right)$ be the choice space on $\{a, b\}$ defined by $\underline{a b}$. The resolution $\left(X, c_{\gtrsim}\right) \oplus_{x}\left(Y, c_{Y}\right)$ is a resolvable choice space, which is also rationalizable but does not satisfy WARNI.

The following final example shows that the dotted area in Fig. 2 is nonempty.

Example 13 (Resolvable and WARNI, but not WARP) Define a weak preference $\succsim$ on $X=\{x, y, z\}$ by letting $x \sim y, x>z$, and $y \sim z$. It is easy to check that $\gtrsim$ is quasitransitive and total, but not transitive. Again, let $c_{\gtrsim}: \Omega_{X} \rightarrow \Omega_{X}$ be the choice correspondence derived from $\gtrsim$, that is, $x y, x z, y z$, and $x y z$. By construction, the choice space $\left(X, c_{\succ}\right)$ is quasi-transitively rationalizable but not transitively rationalizable, hence WAR $\tilde{R}$ N holds and WARP fails by Theorem 'Rationalization by Axioms'. Furthermore, $\left(X, c_{\gtrsim}\right)$ is resolvable by Corollary 4 , because the proper menu $\{x, z\}$ is shrinkable.

\section{Conclusions and future directions of research}

In this paper we have introduced a novel operation for choice spaces, called onepoint resolution. This operation reveals the possibility to decompose a given choice behavior in two simpler choice behaviors. One-point resolutions are fully general for

\footnotetext{
${ }^{23}$ Observe that the derived strict preference $>$ is the $N$-shaped strict partial order on a 4-element set.

${ }^{24}$ In fact, properties $(\alpha)$ and $(\gamma)$ hold, whereas axiom $(\rho)$ fails: indeed, we have $z \in c_{\gtrsim}(\{x, z\})$ and $z \notin c_{\gtrsim}(\{x, y, z\})$, but $y \notin c_{\gtrsim}(\{x, y, z\})$.
} 
deconstruction purposes, because any process of decomposition of a choice behavior can be obtained as a sequential/iterative application of one-point resolutions. Resolutions also have a natural interpretation, since they detect a delegation of tasks to smaller decisional units.

The main results of this paper are of three types: (1) resolutions preserve most axioms of choice consistency, in particular rationality features; (2) resolutions can be characterized by the existence of shrinkable proper menus; (3) resolutions are connected to rationality whenever the relation of revealed preference is transitive.

Future research on the topic goes in three main directions. First, we are currently studying some useful properties ('hereditariness', 'transitivity', etc.) of resolvable spaces and shrinkable menus, which further suggest the naturalness of the operation of resolution. These properties may be useful to derive computationally effective conditions to detect the shrinkability of menus (Cantone et al. 2020a).

A more complex issue is the 'essential uniqueness' of the decomposition of a (finite) choice behavior. One can show that every finite choice can be uniquely expressed as an iterated resolution of choices, in which points are resolved into either preordered choices (i.e., rationalizable by a preorder) or irresolvable choices, and points of a preordered choice are never resolved into a preordered choice. Thus, a finite choice always reveals a unique corporate hierarchy, in which the reasoning by each executive is either 'maximally rational' or 'minimally irrational', but no rational executive reports to another rational executive.

Finally, resolutions are useful in other fields of research, e.g., convex geometries. Recall that a convex geometry (Edelman and Jamison 1985) is a pair $(X, \mathcal{C})$, where $X$ is a nonempty finite set, and $\mathcal{C}$ is a family of subsets of $X$ that contains the empty set, is closed under taking intersections, and is upgradable (i.e., for any $A$ in $\mathcal{C} \backslash\{X\}$, there is $x$ in $X \backslash A$ such that $A \cup\{x\} \in \mathcal{C}$ ). Koshevoy (1999) explicitly gives a structure-preserving bijection between convex geometries on $X$ and path independent choices on $X$. Since, by the results of this paper, any resolution of path independent choices is path independent, a notion of resolution of convex geometries naturally arises: see Cantone et al. (2020b). Notice that the above analysis turns to be useful in other related fields of research, since the notion of convex geometry is 'dual' to those of learning spaces (Falmagne and Doignon 2011), antimatroids (Jamison 1980), and meet-distributive lattices (Edelman 1980). ${ }^{25}$

Acknowledgements The authors wish to thank the handling editor Clemens Puppe and two anonymous referees for their comments and suggestions, which determined a remarkable improvement in the quality of the paper and the focus of the presentation. The authors are also grateful to José C. R. Alcantud, Jean-Paul Doignon, and Efe A. Ok for useful suggestions. The first author acknowledges support by "Università degli Studi di Catania, Piano della Ricerca 2016/2018 Linea di intervento 2". The second author acknowledges support by "Università degli Studi di Catania, FIR-2014 BCAEA3".

Funding Open access funding provided by Università degli Studi di Catania within the CRUI-CARE Agreement.

\footnotetext{
25 See also the pioneering work of Dilworth (1940) on lattices having special properties, as well as the book on greedoids by Korte et al. (1991).
} 
Open Access This article is licensed under a Creative Commons Attribution 4.0 International License, which permits use, sharing, adaptation, distribution and reproduction in any medium or format, as long as you give appropriate credit to the original author(s) and the source, provide a link to the Creative Commons licence, and indicate if changes were made. The images or other third party material in this article are included in the article's Creative Commons licence, unless indicated otherwise in a credit line to the material. If material is not included in the article's Creative Commons licence and your intended use is not permitted by statutory regulation or exceeds the permitted use, you will need to obtain permission directly from the copyright holder. To view a copy of this licence, visit http://creativecommons.org/licen ses/by/4.0/.

\section{Appendix: Proofs}

\section{Section 2 (resolutions)}

Proof of Lemma 1: The first formulation of $c_{Z}(A)$ readily follows from Definition 3 and Lemma 6 below, whose simple proof is left to the reader. The second formulation of $c_{Z}(A)$ is an immediate consequence of the definition of projection.

Lemma 6 Let $\left(Z, c_{Z}\right)=\left(X, c_{X}\right) \oplus_{x}\left(Y, c_{Y}\right)$ be a one-point resolution. For each $A \in \Omega_{Z}$, $\pi(A)=A$ if $A \cap Y=\emptyset$, and $\pi(A)=(A \cap X) \cup\{x\}$ otherwise. Further, for each $B \in 2^{X}, \pi^{-1}(B)=B$ if $x \notin B$, and $\pi^{-1}(B)=(B \backslash\{x\}) \cup Y$ otherwise.

Proof of Lemma 2: Parts (i) and (ii) are reformulations of the first two lines of (4) in Lemma 1. To prove (iii), let $y \in Y$. We shall show that the equality $\pi\left(c_{Z}(A)\right)=c_{X}(\pi(A))$ holds for each menu $A \subseteq(X \backslash\{x\}) \cup\{y\}$. We consider two cases: (1) $y \notin A$; (2) $y \in A$. In case (1), the inclusion $A \subseteq X \backslash\{x\}$ holds and $\pi \uparrow_{A}$ is the identity, hence the claim is true by part (ii). In case (2), assume first that $x \in c_{X}(\pi(A))$. The first line of (3) in Definition 3 yields

$$
\pi\left(c_{Z}(A)\right)=\pi\left(\left(c_{X}(\pi(A)) \backslash\{x\}\right) \cup c_{Y}(\{y\})\right)=\left(c_{X}(\pi(A)) \backslash\{x\}\right) \cup\{x\}=c_{X}(\pi(A))
$$

and so the claim holds. On the other hand, if $x \notin c_{X}(\pi(A))$, then

$$
\pi\left(c_{Z}(A)\right)=\pi\left(c_{X}(\pi(A))\right)=c_{X}(\pi(A))
$$

again by definition. This completes the proof.

Proof of Example 5: For convenience, let us recall the definition of Gemma's extended choice on the set $Z=\{c, m, p, s, t\}=\{$ chips, salmon, pizza, sea bass, tuna $\}$ :

$$
\begin{aligned}
& \text { tmcps, }
\end{aligned}
$$

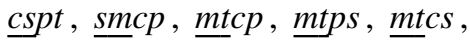

$$
\begin{aligned}
& \underline{c s p}, \underline{c t p}, \underline{p s t}, \underline{c s t}, \underline{m s p}, \underline{m t p}, \bar{p} c m, \underline{m t s}, \underline{m t c}, \underline{c s m} \text {, } \\
& \underline{p c}, \underline{p s}, \underline{p t}, \underline{c s}, \underline{c t}, \underline{s t}, \underline{p m}, \underline{m c}, \underline{m s}, \underline{m t} .
\end{aligned}
$$

We claim that this choice is irresolvable. To prove the claim, we use some easy results, which substantially simplify computations. Indeed, it can be shown that the computational complexity of determining whether a choice is resolvable is 
exponential. In practice, however, computations can often be expedited in polynomial time, by quickly eliminating many possible decompositions. This is exactly what we shall do in our example, determining the irresolvability of Gemma's extended choice by applying the following simple rules (all of which run in polynomial time):

Lemma 7 Let $\left(Z, c_{Z}\right)$ be a choice space, and $x, y$, zitems in $Z$ such that any of the following four cases happens: (1) $\underline{x z}$ and $y \underline{z}$, or (2) $\underline{x} z$ and $y \underline{z}$, or (3) $x \underline{z}$ and $\underline{x y z}$, or (4) xy and xyz. For any candidate fiber space $\left(Y, c_{Y}\right)$, if $x, y \in \bar{Y} Y$, then $z \bar{\epsilon} \in Y$.

Proof Let $x, y, z \in Z$ be such that any among the four cases 1-4 happens. Toward a contradiction, suppose $\left(Z, c_{Z}\right)=\left(X, c_{X}\right) \oplus_{x_{0}}\left(Y, c_{Y}\right), x, y \in Y$, and $z \in X$. Assume that case 1 happens. By the definition of resolution, $c_{Z}(\{x, z\})=\{x\}$ implies $z \notin c_{X}\left(\left\{z, x_{0}\right\}\right)=\left\{x_{0}\right\}$, whereas $c_{Z}(\{y, z\})=\{y, z\}$ implies $z \in c_{X}\left(\left\{z, x_{0}\right\}\right)=\left\{z, x_{0}\right\}$, a contradiction. Case 2 is handled similarly. For case $3, c_{Z}(\{x, z\})=\{z\}$ implies $c_{X}\left(\left\{z, x_{0}\right\}\right)=\{z\}, \quad$ whereas $\quad c_{Z}(\{x, y, z\})=\{x, y\} \quad$ implies $\quad c_{X}\left(\left\{z, x_{0}\right\}\right)=\left\{x_{0}\right\}$ (and $\left.c_{Y}(\{x, y\})=\{x, y\}\right)$, a contradiction. For case 4, $c_{Z}(\{x, y\})=\{x\}$ implies $c_{Y}(\{x, y\})=\{x\}$, whereas $c_{Z}(\{x, y, z\})=\{y, z\}$ implies $c_{Y}(\{x, y\})=\{y\} \quad$ (and $\left.c_{X}\left(\left\{z, x_{0}\right\}\right)=\left\{z, x_{0}\right\}\right)$, a contradiction.

Observe that checking rules (1) and (2) in Lemma 7 takes quadratic time, while checking rules (3) and (4) takes cubic time. In practice, a computer could first check (1) and (2), and then (3) and (4). Using the four rules in Lemma 7, we can quickly conclude that Gemma's extended choice is irresolvable. Indeed, the possible fiber spaces are $25=\left(2^{5}-1\right)-5-1$. An application of rule (1) rules out 19 of them, leaving only the sets $\{c, t\},\{s, t\},\{c, m\},\{c, s, t\},\{c, m, t\}$, and $\{c, m, s, t\}{ }^{26}$ Then an application of rules (3) and (4) eliminates the remaining 6 possible fiber spaces as well (computations are left to the reader).

Proof of Theorem 1(ii): The following 'intermediate' version of the notion of resolution, which encompasses both one-point and horizontal resolutions, will be needed:

Definition 11 Let $\left(X, c_{X}\right)$ be a choice space, and $\left(Y_{x}, c_{x}\right)_{x \in V}$ a family of choice spaces indexed by $V \subseteq X$. Set $Z_{V}:=(X \backslash V) \cup \bigcup_{x \in V} Y_{x}$. Define the projection $\pi_{V}: Z_{V} \rightarrow X$ by

$$
\pi_{V}(z):= \begin{cases}z & \text { if } z \in X \backslash V \\ x & \text { if } z \in Y_{x} \text { for some } x \in V .\end{cases}
$$

Finally, define a choice correspondence $c_{Z_{V}}$ on $Z_{V}$ by setting, for each $A \in 2^{Z_{V}}$,

\footnotetext{
${ }^{26}$ For instance, having $Y=\{p, s\}$ as fiber set is impossible, since $p c$ and $s c$ imply $c \in Y$, a contradiction. Similarly, we cannot have $Y=\{m, p, t\}$, since $p s$ and $\underline{m s}$ imply $\bar{s} \in Y$, a contradiction. Furthermore, $Y=\{c, p, s, t\}$ is impossible, since $p m$ and $\underline{s m}$ imply $m \in Y$, a contradiction. And so on.
} 


$$
c_{Z_{V}}(A):=\bigcup_{x \in c_{X}\left(\pi_{V}(A)\right) \cap V} c_{x}\left(A \cap Y_{x}\right) \cup\left(c_{X}\left(\pi_{V}(A)\right) \backslash V\right) .
$$

Then $\left(Z_{V}, c_{Z_{V}}\right)=\left(X, c_{X}\right) \oplus\left(Y_{x}, c_{x}\right)_{x \in V}$ is the partial resolution of $\left(X, c_{X}\right)$ into $\left(Y_{x}, c_{x}\right)_{x \in V}$.

The notation for partial resolutions is unambiguous, as the next remark shows.

Remark 3 (i) If $V=X$, then the partial resolution $\left(Z_{V}, c_{Z_{V}}\right)=\left(X, c_{X}\right) \oplus\left(Y_{x}, c_{x}\right)_{x \in V}$ is just the horizontal resolution $\left(Z, c_{Z}\right)=\left(X, c_{X}\right) \oplus\left(Y_{x}, c_{x}\right)_{x \in X}$.

(ii) If $V=\{a\}$, with $a \in X$, then the partial resolution $\left(X, c_{X}\right) \oplus\left(Y_{x}, c_{x}\right)_{x \in V}$ is the one-point resolution $\left(X, c_{X}\right) \oplus_{a}\left(Y_{a}, c_{a}\right)$.

(iii) Finally, if $V=\emptyset$, then we simply have $\left(X, c_{X}\right) \oplus\left(Y_{x}, c_{x}\right)_{x \in V}=\left(X, c_{X}\right)$.

The key step in the proof of Theorem 1(ii) is the most technical result of the paper:

Lemma 8 (Decomposition Lemma) Let $\left(X, c_{X}\right)$ be a choice space, $\bar{x}$ a point in $X$, and $\left(Y_{x}, c_{x}\right)_{x \in X}$ a family of pairwise disjoint choice spaces disjoint from $(X, c)$. We have:

$$
\left(X, c_{X}\right) \oplus\left(Y_{x}, c_{x}\right)_{x \in X}=\left(\left(X, c_{X}\right) \oplus\left(Y_{x}, c_{x}\right)_{x \in X \backslash\{\bar{x}\}}\right) \oplus_{\bar{x}}\left(Y_{\bar{x}}, c_{\bar{x}}\right)
$$

Proof Let $\left(Z, c_{Z}\right)$ be the horizontal resolution of $\left(X, c_{X}\right)$ into $\left(Y_{x}, c_{x}\right)_{x \in X}$, i.e.,

$$
\left(Z, c_{Z}\right):=\left(X, c_{X}\right) \oplus\left(Y_{x}, c_{x}\right)_{x \in X}
$$

Therefore, $Z=\bigcup_{x \in X} Y_{x}$, and, for any $A \in 2^{Z}$,

$$
c_{Z}(A):=\bigcup_{x \in c_{X}(\pi(A))} c_{x}\left(A \cap Y_{x}\right)
$$

where $\pi: Z \rightarrow X$ is the projection of $Z$ over $X$ satisfying $z \in Y_{\pi(z)}$, for every $z \in Z$. Furthermore, for a given distinguished point $\bar{x}$ of $X$, let

$$
\left(Z^{\prime}, c_{Z^{\prime}}\right):=\left(X, c_{X}\right) \oplus\left(Y_{x}, c_{x}\right)_{x \in X \backslash\{\bar{x}\}}
$$

Hence, setting for $x \in X$

$$
Y_{x}^{\prime}:= \begin{cases}Y_{x} & \text { if } x \neq \bar{x} \\ \{\bar{x}\} & \text { if } x=\bar{x}\end{cases}
$$

we obtain $Z^{\prime}:=\bigcup_{x \in X} Y_{x}^{\prime}=\bigcup_{x \in X \backslash\{\bar{x}\}} Y_{x} \cup\{\bar{x}\}$. We also have, for any $A^{\prime}$ in $2^{Z^{\prime}}$,

$$
c_{Z^{\prime}}\left(A^{\prime}\right)=\bigcup_{x \in c_{X}\left(\pi^{\prime}\left(A^{\prime}\right)\right)} c_{x}^{\prime}\left(A^{\prime} \cap Y_{x}^{\prime}\right),
$$

where $\pi^{\prime}: Z^{\prime} \rightarrow X$ is the projection satisfying $z \in Y_{\pi^{\prime}(z)}^{\prime}$ for every $z \in Z^{\prime}$, and, for $x \in X$, 


$$
c_{x}^{\prime}:= \begin{cases}c_{x} & \text { if } x \neq \bar{x} \\ \text { the choice over }\{\bar{x}\} & \text { if } x=\bar{x}\end{cases}
$$

Finally, let $\left(Z, \bar{c}_{Z}\right)$ be the resolution of $\left(Z^{\prime}, c_{Z^{\prime}}\right)$ at $\bar{x}$ into $\left(Y_{\bar{x}}, c_{\bar{x}}\right)$, i.e.,

$$
\left(Z, \bar{c}_{Z}\right):=\left(\left(X, c_{X}\right) \oplus\left(Y_{x}, c_{x}\right)_{x \in X \backslash\{\bar{x}\}}\right) \oplus_{\bar{x}}\left(Y_{\bar{x}}, c_{\bar{x}}\right)
$$

Thus, for $A \in 2^{Z}$, we have

$$
\bar{c}_{Z}(A)= \begin{cases}\left(c_{Z^{\prime}}(\bar{\pi}(A)) \backslash\{\bar{x}\}\right) \cup c_{\bar{x}}\left(A \cap Y_{\bar{x}}\right) & \text { if } \bar{x} \in c_{Z^{\prime}}(\bar{\pi}(A)) \\ c_{Z^{\prime}}(\bar{\pi}(A)) & \text { otherwise, }\end{cases}
$$

where $\bar{\pi}: Z \rightarrow Z^{\prime}$ is the projection of $Z$ over $Z^{\prime}$, defined as follows for each $z \in Z$ :

$$
\bar{\pi}(z):=\left\{\begin{array}{l}
z \text { if } z \notin Y_{\bar{x}} \\
\bar{x} \text { if } z \in Y_{\bar{x}}
\end{array}\right.
$$

In view of (7) and (8), our task has thus been reduced to proving $\left(Z, \bar{c}_{Z}\right)=\left(Z, c_{Z}\right)$, i.e.,

$$
c_{Z}=\bar{c}_{Z}
$$

To that end, we need some technical facts, stated by the next two lemmas.

Lemma $9 \pi^{\prime} \circ \bar{\pi}=\pi$.

Proof Let $z \in Z=\bigcup_{x \in X} Y_{x}$. Then, we have:

$$
\pi^{\prime}(\bar{\pi}(z))=\left\{\begin{array}{ll}
\pi^{\prime}(z) & \text { if } z \notin Y_{\bar{x}} \\
\pi^{\prime}(\bar{x}) & \text { if } z \in Y_{\bar{x}}
\end{array}=\left\{\begin{array}{ll}
\pi(z) & \text { if } z \notin Y_{\bar{x}} \\
\bar{x} & \text { if } z \in Y_{\bar{x}}
\end{array}=\pi(z),\right.\right.
$$

which proves the claim.

To enhance clarity, in what follows we shall make use of conditional expressions (borrowed from the $\mathrm{C}$ programming language), namely expressions of type $[$ Cond $? a: b]$, defined by

$$
[\text { Cond } ? a: b]:=\left\{\begin{array}{l}
a \text { if Cond holds } \\
b \text { if Cond does not hold. }
\end{array}\right.
$$

Lemma 10 For $A \in 2^{Z}$, we have:

(a) $\bar{x} \in c_{Z^{\prime}}(\bar{\pi}(A)) \Longleftrightarrow \bar{x} \in c_{X}(\pi(A))$;

(b) if $x \in X \backslash\{\bar{x}\}$, then $\bar{\pi}(A) \cap Y_{x}=A \cap Y_{x}$;

(c) $c_{Z^{\prime}}(\bar{\pi}(A)) \backslash\{\bar{x}\}=\bigcup_{x \in c_{X}(\pi(A)), x \neq \bar{x}} c_{x}\left(A \cap Y_{x}\right)$.

Proof Let $A \in 2^{Z}$. For (a), we have: 


$$
\begin{aligned}
\bar{x} \in c_{Z^{\prime}}(\bar{\pi}(A)) & \Longleftrightarrow \bar{x} \in \bigcup_{x \in c_{X}\left(\pi^{\prime}(\bar{\pi}(A))\right)} c_{x}^{\prime}\left(\bar{\pi}(A) \cap Y_{x}^{\prime}\right) \\
& \Longleftrightarrow \bar{x} \in \bigcup_{x \in c_{X}(\pi(A))} c_{x}^{\prime}\left(\bar{\pi}(A) \cap Y_{x}^{\prime}\right) \\
& \Longleftrightarrow \bar{x} \in c_{X}(\pi(A)) \wedge \bar{x} \in c_{\bar{x}}^{\prime}\left(\bar{\pi}(A) \cap Y_{\bar{x}}^{\prime}\right) \\
& \Longleftrightarrow \bar{x} \in c_{X}(\pi(A)) \wedge \bar{x} \in c_{\bar{x}}^{\prime}(\bar{\pi}(A) \cap\{\bar{x}\}) \\
& \Longleftrightarrow \bar{x} \in c_{X}(\pi(A)),
\end{aligned}
$$

where the last equivalence is justified by the chain of the following implications:

$$
\begin{aligned}
\bar{x} \in c_{X}(\pi(A)) & \Longrightarrow A \cap Y_{\bar{x}} \neq \emptyset \\
& \Longrightarrow \bar{x} \in \bar{\pi}(A) \\
& \Longrightarrow \bar{x} \in c_{\bar{x}}^{\prime}(\bar{\pi}(A) \cap\{\bar{x}\}) .
\end{aligned}
$$

For (b), since $\bar{\pi}(A)=\left(A \backslash Y_{\bar{x}}\right) \cup\left[A \cap Y_{\bar{x}} \neq \emptyset ?\{\bar{x}\}: \emptyset\right]$, for $x \in X \backslash\{\bar{x}\}$ we have:

$$
\begin{aligned}
\bar{\pi}(A) \cap Y_{x} & =\left(\left(A \backslash Y_{\bar{x}}\right) \cup\left[A \cap Y_{\bar{x}} \neq \emptyset ?\{\bar{x}\}: \emptyset\right]\right) \cap Y_{x} \\
& =\left(A \backslash Y_{\bar{x}}\right) \cap Y_{x} \\
& =A \cap Y_{x} .
\end{aligned}
$$

Finally, concerning (c), we have:

$$
\begin{aligned}
c_{Z^{\prime}}(\bar{\pi}(A)) & =\bigcup_{x \in c_{X}\left(\pi^{\prime}(\bar{\pi}(A))\right)} c_{x}^{\prime}\left(\bar{\pi}(A) \cap Y_{x}^{\prime}\right) \\
& =\bigcup_{x \in c_{X}(\pi(A))} c_{x}^{\prime}\left(\bar{\pi}(A) \cap Y_{x}^{\prime}\right) \\
& =\bigcup_{x \in c_{X}(\pi(A)), x \neq \bar{x}} c_{x}^{\prime}\left(\bar{\pi}(A) \cap Y_{x}^{\prime}\right) \cup\left[\bar{x} \in c_{X}(\pi(A)) ? c_{\bar{x}}^{\prime}\left(\bar{\pi}(A) \cap Y_{\bar{x}}^{\prime}\right): \emptyset\right] \\
& =\bigcup_{x \in c_{X}(\pi(A)), x \neq \bar{x}} c_{x}\left(A \cap Y_{x}\right) \cup\left[\bar{x} \in c_{X}(\pi(A)) ?\{\bar{x}\}: \emptyset\right],
\end{aligned}
$$

where the last equality follows from (10) and the equality $c_{\bar{x}}^{\prime}\left(\bar{\pi}(A) \cap Y_{\bar{x}}^{\prime}\right)=\{\bar{x}\}$ (provided that $\bar{x} \in c_{X}(\pi(A))$. Since

$$
\bar{x} \notin \bigcup_{x \in c_{X}(\pi(A)), x \neq \bar{x}} c_{x}\left(A \cap Y_{x}\right) \quad \text { and } \quad\left[\bar{x} \in c_{X}(\pi(A)) ?\{\bar{x}\}: \emptyset\right] \subseteq\{\bar{x}\},
$$

it follows that $c_{Z^{\prime}}(\bar{\pi}(A)) \backslash\{\bar{x}\}=\bigcup_{x \in c_{X}(\pi(A)), x \neq \bar{x}} c_{x}\left(A \cap Y_{x}\right)$, which proves (c).

We are now ready to prove Equation (9).

Lemma $11 c_{Z}=\bar{c}_{Z}$.

Proof Let $A \in 2^{Z}$. Then we have: 


$$
\begin{aligned}
c_{Z}(A) & =\bigcup_{x \in c_{X}(\dot{\pi}(A))} c_{x}\left(A \cap Y_{x}\right) \\
& =\bigcup_{x \in c_{X}(\pi(A)), x \neq \bar{x}} c_{x}\left(A \cap Y_{x}\right) \cup\left[\bar{x} \in c_{X}(\pi(A)) ? c_{\bar{x}}\left(A \cap Y_{\bar{x}}\right): \emptyset\right]
\end{aligned}
$$

and

$$
\bar{c}_{Z}(A)=\left(c_{Z^{\prime}}(\bar{\pi}(A)) \backslash\{\bar{x}\}\right) \cup\left[\bar{x} \in c_{Z^{\prime}}(\bar{\pi}(A)) ? c_{\bar{x}}\left(A \cap Y_{\bar{x}}\right): \emptyset\right] .
$$

By Lemma 10(a), $\bar{x} \in c_{Z^{\prime}}(\bar{\pi}(A)) \Longleftrightarrow \bar{x} \in c_{X}(\pi(A))$. It follows that

$$
\left[\bar{x} \in c_{X}(\pi(A)) ? c_{\bar{x}}\left(A \cap Y_{\bar{x}}\right): \emptyset\right]=\left[\bar{x} \in c_{Z^{\prime}}(\bar{\pi}(A)) ? c_{\bar{x}}\left(A \cap Y_{\bar{x}}\right): \emptyset\right] .
$$

In addition, Lemma (10)(c) yields

$$
c_{Z^{\prime}}(\bar{\pi}(A)) \backslash\{\bar{x}\}=\bigcup_{x \in c_{X}(\pi(A)), x \neq \bar{x}} c_{x}\left(A \cap Y_{x}\right) .
$$

This proves Lemma 11.

Now the Decomposition Lemma easily follows from (7), (8), and Lemma 11.

The main consequence of the Decomposition Lemma is what we were after:

Lemma 12 (Permutation Invariance of Resolutions) Let $\left(X, c_{X}\right)$ be a finite choice space, with $X=\left\{x_{1}, \ldots, x_{k}\right\}$. Further, let $\left(Y_{x}, c_{x}\right)_{x \in X}$ be a family of choice spaces, whose ground sets are pairwise disjoint and disjoint from $X$. Then, we have:

$$
\left(X, c_{X}\right) \oplus\left(Y_{x}, c_{x}\right)_{x \in X}=\left(\cdots\left(\left(\left(X, c_{X}\right) \oplus_{x_{1}}\left(Y_{x_{1}}, c_{x_{1}}\right)\right) \oplus_{x_{2}}\left(Y_{x_{2}}, c_{x_{2}}\right)\right) \cdots\right) \oplus_{x_{k}}\left(Y_{x_{k}}, c_{x_{k}}\right)
$$

Proof Let $\left(X, c_{X}\right)$ and $\left(Y_{x}, c_{x}\right)_{x \in X}$ be as in the hypothesis. We proceed by induction on $k=|X|$. For $k=1$, we plainly have $\left(X, c_{X}\right) \oplus\left(Y_{x}, c_{x}\right)_{x \in X}=\left(X, c_{X}\right) \oplus_{x_{1}}\left(Y_{x_{1}}, c_{x_{1}}\right)$. For the inductive step, let $k>1$. A sequential application of Lemma 8 and the inductive hypothesis yields

$$
\begin{aligned}
\left(X, c_{X}\right) \oplus\left(Y_{x}, c_{x}\right)_{x \in X} & =\left(\left(X, c_{X}\right) \oplus\left(Y_{x}, c_{x}\right)_{x \in X \backslash\left\{x_{k}\right\}}\right) \oplus_{x_{k}}\left(Y_{x_{k}}, c_{x_{k}}\right) \\
& =\left(\left(\cdots\left(\left(X, c_{X}\right) \oplus_{x_{1}}\left(Y_{x_{1}}, c_{x_{1}}\right)\right) \oplus_{x_{2}} \cdots\right) \oplus_{x_{k-1}}\left(Y_{x_{k-1}}, c_{x_{k-1}}\right)\right) \oplus_{x_{k}}\left(Y_{x_{k}}, c_{x_{k}}\right)
\end{aligned}
$$

as required.

\section{Section 3 (consistency of resolutions)}

Proof of Theorem 2: The forward part of the equivalence follows at once from Lemma 3. Next, we show that all reverse implications hold as well. Let $\left(Z, c_{Z}\right)=\left(X, c_{X}\right) \oplus_{x}\left(Y, c_{Y}\right)$ be a one-point resolution, and $\pi$ the projection. Let us recall the definition of $c_{Z}$ for each $A \in \Omega_{Z}$ : 


$$
c_{Z}(A)= \begin{cases}\left(c_{X}(\pi(A)) \backslash\{x\}\right) \cup c_{Y}(A \cap Y) & \text { if } x \in c_{X}(\pi(A)) \\ c_{X}(\pi(A)) & \text { otherwise. }\end{cases}
$$

Axiom $(\alpha)$. Suppose $(\alpha)$ holds for both $\left(X, c_{X}\right)$ and $\left(Y, c_{Y}\right)$. Let $z \in Z$ and $A, B \in \Omega_{Z}$ be such that $z \in A \subseteq B$ and $z \in c_{Z}(B)$. We shall show that $z \in c_{Z}(A)$.

Case1 : Suppose $z \in X \backslash\{x\}$. By (10), the hypothesis $z \in c_{Z}(B)$ yields $z \in c_{X}(\pi(B))$. Since $z \in A$ and $(\alpha)$ holds for $\left(X, c_{X}\right)$, we get $z \in c_{X}(\pi(A))$, which in turn implies $z \in c_{Z}(A)$.

Case2 : Suppose $z \in Y$. By (10), we have $x \in c_{X}(\pi(B))$ and $z \in c_{Y}(B \cap Y)$. Since

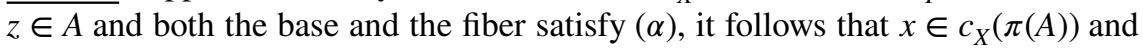
$z \in c_{Y}(A \cap Y)$. Thus, we conclude that $z \in c_{Z}(A)$ holds also in this case.

Axiom $(\gamma)$. Suppose $(\gamma)$ holds for both $\left(X, c_{X}\right)$ and $\left(Y, c_{Y}\right)$. Let $z \in Z$ and $\left\{A_{i}: i \in I\right\} \subseteq \Omega_{Z}$ be such that $z \in \bigcap_{i \in I} c_{Z}\left(A_{i}\right)$. We shall show that $z \in c_{Z}\left(\bigcup_{i \in I} A_{i}\right)$.

Case1 : Suppose $z \in X \backslash\{x\}$. For each $i \in I, z \in c_{Z}\left(A_{i}\right)$ implies $z \in c_{X}\left(\pi\left(A_{i}\right)\right)$. Since $\bigcup_{i \in I} \pi\left(A_{i}\right)=\pi\left(\bigcup_{i \in I} A_{i}\right)$, and $\left(X, c_{X}\right)$ satisfies $(\gamma)$, it follows that $z \in c_{X}\left(\bigcup_{i \in I} \pi\left(A_{i}\right)\right)=c_{X}\left(\pi\left(\bigcup_{i \in I} A_{i}\right)\right)$. Thus, we get $z \in c_{Z}\left(\bigcup_{i \in I} A_{i}\right)$, as wanted.

Case2: Suppose $z \in Y$. The first line of (10) yields $x \in c_{X}\left(\pi\left(A_{i}\right)\right)$ and $z \in c_{Y}\left(A_{i} \cap Y\right)$ for each $i \in I$. Since both the base and the fiber satisfy $(\gamma)$, we get $x \in c_{X}\left(\bigcup_{i \in I}\left(\pi\left(A_{i}\right)\right)\right)=c_{X}\left(\pi\left(\bigcup_{i \in I} A_{i}\right)\right) \quad$ and $z \in c_{Y}\left(\bigcup_{i \in I}\left(A_{i} \cap Y\right)\right)=c_{Y}\left(\bigcup_{i \in I} A_{i} \cap Y\right)$. Thus, we conclude that $z \in c_{Z}\left(\bigcup_{i \in I} A_{i}\right)$ holds also in this case.

Axiom $(\rho)$. Suppose $(\rho)$ holds for $\left(X, c_{X}\right)$ and $\left(Y, c_{Y}\right)$. Let $w, z \in Z$ and $A \in \Omega_{Z}$ be such that $w \in c_{Z}(A)$ and $w \notin c_{Z}(A \cup\{z\})$. We shall show that $z \in c_{Z}(A \cup\{z\})$.

Case1 : Suppose $w, z \in X \backslash\{x\}$. The hypothesis yields $w \in c_{X}(\pi(A)) \backslash c_{X}(\pi(A)$ $\cup\{z\})$. Since $(\rho)$ holds for $\left(X, c_{X}\right)$, we get $z \in c_{X}(\pi(A) \cup\{z\})$, hence $z \in c_{Z}(A \cup\{z\})$ by (10), as claimed.

Case2 : Suppose $w, z \in Y$. Since $w \in A \cap Y$, we have $\pi(A)=\pi(A \cup\{z\})=(A \cap X)$ $\cup\{x\}$. By (10), we obtain $x \in c_{X}(\pi(A))=c_{X}(\pi(A \cup\{z\}))$ and $w \in c_{Y}$ $(A \cap Y) \backslash c_{Y}((A \cup\{z\}) \cap Y)$. Since $(\rho)$ holds for $\left(Y, c_{Y}\right)$, we get $z \in c_{Y}((A \cup\{z\}) \cap Y)$, and so (10) yields $z \in c_{Z}(A \cup\{z\})$.

Case3 : Suppose $w \in X \backslash\{x\}$ and $z \in Y$. The hypothesis $w \in c_{Z}(A)$ implies $\overline{w \in c_{X}}(\pi(A))$, whereas the hypothesis $w \notin c_{Z}(A \cup\{z\})$ implies $w \notin c_{X}(\pi(A \cup\{z\}))$. In particular, we must have $\pi(A) \subsetneq \pi(A \cup\{z\})$, which yields $A \subseteq X \backslash\{x\}$, $\pi(A)=A$, and $\pi(A \cup\{z\})=\pi(A) \cup\{x\}=A \cup\{x\}$. Then we can rewrite the previous conclusions as $w \in c_{X}(A)$ and $w \notin c_{X}(A \cup\{x\})$. Since $(\rho)$ holds for $\left(X, c_{X}\right)$, we obtain $x \in c_{X}(A \cup\{x\})=c_{X}(\pi(A \cup\{z\}))$. Further, since $A \subseteq X$, we get 
$(A \cup\{z\}) \cap Y=\{z\}$, and so $z \in c_{Y}(\{z\})=c_{Y}((A \cup\{z\}) \cap Y)$. The latter fact yields that $z \in c_{Z}(A \cup\{z\})$, as claimed.

Case4 : Suppose $w \in Y$ and $z \in X \backslash\{x\}$.

The hypothesis $w \in c_{Z}(A)$ implies $x \in c_{X}(\pi(A))$ and $w \in c_{Y}(A \cap Y)$. Further, since $c_{Y}((A \cup\{z\}) \cap Y)=c_{Y}(A \cap Y)$, the hypothesis $w \notin c_{Z}(A \cup\{z\})$ implies $x \notin c_{X}(\pi(A \cup\{z\}))$. Since $(\rho)$ holds for $\left(X, c_{X}\right)$, it follows that $z \in c_{X}(\pi(A \cup\{z\})$, and so $z \in c_{Z}(A \cup\{z\})$ again.

Proof of Theorem 3: We need a characterization of path independence, based on

$\checkmark$ Axiom ( $(\varepsilon)$ : for all $A, B \in \Omega_{X}$, if $A \subseteq B$, then it is not the case that $c(B) \subsetneq c(A)$.

Then, we have:

Lemma 13 (Blair 1974) PI is equivalent to $(\alpha) \&(\varepsilon)$.

The necessity in Theorem 3 is an immediate consequence of Lemma 3. To prove sufficiency, let $\left(Z, c_{Z}\right)=\left(X, c_{X}\right) \oplus_{x}\left(Y, c_{Y}\right)$ be a resolution and $\pi$ the projection. Suppose $\left(X, c_{X}\right)$ and $\left(Y, c_{Y}\right)$ satisfy $\mathrm{Pl}$, hence, by Lemma 13, $(\alpha)$ and $(\varepsilon)$. Theorem 2 readily yields that $\left(Z, c_{Z}\right)$ satisfies $(\alpha)$. Thus, to complete the proof, it is enough to show that axiom $(\varepsilon)$ holds for $\left(Z, c_{Z}\right)$, too. To that end, we prove that $c_{Z}(B)=c_{Z}(A)$ for all $A, B \in \Omega_{Z}$ such that $A \subseteq B$ and $c_{Z}(B) \subseteq c_{Z}(A)$. The definition of $c_{Z}$ readily yields $c_{X}(\pi(B)) \backslash\{x\} \subseteq c_{X}(\pi(A)) \backslash\{x\}$. Observe that, if $x \in c_{X}(\pi(B))$, then $B \cap Y \neq \emptyset$, hence $c_{Z}(A) \cap Y \supseteq c_{Y}(B \cap Y) \neq \emptyset$, and so $x \in c_{X}(\pi(A))$. It follows that

$$
c_{X}(\pi(B)) \subseteq c_{X}(\pi(A)) .
$$

From $A \subseteq B$, we get $\pi(A) \subseteq \pi(B)$, and so, since $(\varepsilon)$ holds for $\left(X, c_{X}\right)$, (11) implies

$$
c_{X}(\pi(B))=c_{X}(\pi(A)) .
$$

If $x \notin c_{X}(\pi(A))$, then (12) readily implies $c_{Z}(B)=c_{Z}(A)$, again by the definition of $c_{Z}$. On the other hand, if $x \in c_{X}(\pi(A))$, then $x \in c_{X}(\pi(B))$, and so $c_{Y}(B \cap Y) \subseteq c_{Y}(A \cap Y)$. Since $A \cap Y \subseteq B \cap Y$ and $(\varepsilon)$ holds for $\left(Y, c_{Y}\right)$, we get

$$
c_{Y}(B \cap Y)=c_{Y}(A \cap Y) .
$$

Now equations (12) and (13) imply $c_{Z}(B)=c_{Z}(A)$, which completes the proof.

Proof of Theorem 4: Since the one-point resolution, the base and the fiber all satisfy CWDE by hypothesis, we can prove the claim by using the characterization of 2-sequential rationalizability given in Lemma 4.

Necessity is an immediate consequence of Lemmas 3 and 4, because the base and the fiber satisfy CWDE by hypothesis, whereas $(\gamma)$ and WWARP are hereditary properties. 
For sufficiency, suppose the base $\left(X, c_{X}\right)$ and the fiber $\left(Y, c_{Y}\right)$ are 2-sequentially rationalizable. Thus, by Lemma 4 , properties $(\gamma)$ and WWARP hold for both of them. In what follows, we show that $\left(Z, c_{Z}\right)$ satisfies these properties as well, hence another application of Lemma 4 will yield the claim. Recall the definition of $c_{Z}$ for each $A \in \Omega_{Z}$ :

$$
c_{Z}(A)= \begin{cases}\left(c_{X}(\pi(A)) \backslash\{x\}\right) \cup c_{Y}(A \cap Y) & \text { if } x \in c_{X}(\pi(A)) \\ c_{X}(\pi(A)) & \text { otherwise. }\end{cases}
$$

Since axiom $(\gamma)$ holds by Theorem 2, it suffices to show that $\left(Z, c_{Z}\right)$ satisfies WWARP. Thus, let $w, z \in Z$ and $A, B \in \Omega_{Z}$ be such that $w, z \in A \subseteq B$. Assume that $c_{Z}(\{w, z\})=\{w\}$ and $w \in c_{Z}(B)$. Below we show that $z \notin c_{Z}(A)$ in all possible cases.

Case 1: $w, z \in X \backslash\{x\}$. By hypothesis, $w=c_{Z}(\{w, z\})=c_{X}(\{w, z\})$. Since $w \in c_{Z}(B) \cap X$, we also have $w \in c_{X}(\pi(B))$. Since $w, z \in \pi(A) \subseteq \pi(B)$, an application of WWARP in the base space $\left(X, c_{X}\right)$ readily yields $z \notin c_{X}(\pi(A))$, whence $z \notin c_{Z}(A)$ by (14), as claimed.

Case 2: $w, z \in Y$. By an argument similar to that of Case 1, we obtain $c_{Y}(\{w, z\})=\{w\}$ and $w \in c(B \cap Y)$. Thus, an application of WWARP in the fiber space $\left(Y, c_{Y}\right)$ entails $z \notin c_{Y}(A \cap Y)$, which implies $z \notin c_{Z}(A)$, as required.

Case 3: $w \in X \backslash\{x\}$ and $z \in Y$. The hypothesis $c_{Z}(\{w, z\})=\{w\}$ implies $x \notin c_{X}(\pi(\{w, z\}))=c_{X}(\{w, x\})$, hence $c_{X}(\{w, x\})=\{w\}$. Moreover, $w \in c_{Z}(B)$ yields $w \in c_{X}(\pi(B))$. An application of WWARP in $\left(X, c_{X}\right)$ gives $x \notin c_{X}(\pi(A))$, whence $c_{Z}(A)=c_{X}(\pi(A))$ by definition (14). Since $z \in Y$, it follows that $z \notin c_{Z}(A)$, as claimed.

Case 4: $w \in Y$ and $z \in X \backslash\{x\}$. Since $c_{Z}(\{w, z\})=\{w\}$, we get $x \in c_{X}(\pi(\{w, z\}))=c_{X}(\{x, z\})$. Further, we have $z \notin c_{X}(\{x, z\})$, since otherwise we would get $c_{Z}(\{w, z\})=\{w, z\}$, a contradiction. It follows that $c_{X}(\{x, z\})=\{x\}$. Moreover, since $w \in c_{Z}(B) \cap Y$, we have $x \in c_{X}(\pi(B))$. By WWARP in $\left(X, c_{X}\right)$, we get $z \notin c_{X}(\pi(A))$, and so $z \notin c_{Z}(A)$ again.

This completes the proof of Theorem 4.

Proof of Theorem 5: We can assume that $\left(Z, c_{Z}\right)=\left(X, c_{X}\right) \oplus_{x}\left(Y, c_{Y}\right)$ is nontrivial.

$(i) \Rightarrow(i i)$. Suppose the one-point resolution $\left(Z, c_{Z}\right)=\left(X, c_{X}\right) \oplus_{x}\left(Y, c_{Y}\right)$ satisfies axiom $(\beta)$. Since $(\beta)$ is a hereditary property, Lemma 3 implies that $(\beta)$ holds for both $\left(X, c_{X}\right)$ and $\left(Y, c_{Y}\right)$. To complete the proof of this implication, it remains to show that either $x$ is a repellent point of $\left(X, c_{X}\right)$ or $c_{Y}$ is the identity map. Toward a contradiction, assume that neither holds. Thus, there exist $S \subseteq X$ such that $\{x\} \subsetneq c_{X}(S)$, and $T \subseteq Y$ such that $c_{Y}(T) \subsetneq T$. Choose $s \in c_{X}(S) \backslash\{x\}$ and $t \in T \backslash c_{Y}(T)$, and set 


$$
A=(S \backslash\{x\}) \cup\{t\} \quad \text { and } \quad B=(S \backslash\{x\}) \cup T .
$$

Notice that $A$ and $B$ intersect both $X$ and $Y$, and

$$
\pi(A)=(A \cap X) \cup\{x\}=S, \quad \pi(B)=(B \cap X) \cup\{x\}=S, \quad A \cap Y=\{t\}, \quad B \cap Y=T,
$$

where $\pi$ is the projection associated with $\left(Z, c_{Z}\right)$. Since $x \in c_{X}(S)=c_{X}(\pi(A))=c_{X}(\pi(B))$, the fourth line of (10) in Lemma 1 yields

$c_{Z}(A)=\left(c_{X}(S) \backslash\{x\}\right) \cup\{t\} \quad$ and $\quad c_{Z}(B)=\left(c_{X}(S) \backslash\{x\}\right) \cup c_{Y}(T)$.

Then we conclude that $A$ and $B$ are menus in $\left(Z, c_{Z}\right)$ such that $A \subseteq B, s, t \in c_{Z}(A)$, $s \in c_{Z}(B)$, and $t \notin c_{Z}(B)$. However, this contradicts the fact that $(\beta)$ holds for $\left(Z, c_{Z}\right)$.

(ii) $\Rightarrow($ i). Assume that (ii) holds. To start, we prove the following property:

Lemma 14 Let $\left(Z, c_{Z}\right)=\left(X, c_{X}\right) \oplus_{x}\left(Y, c_{Y}\right)$ be a one-point resolution. For any menu $A \in 2^{Z}$ and item $z \in c_{Z}(A)$, we have $\pi(z) \in c_{X}(\pi(A))$, where $\pi$ is the projection.

Proof Let $A \in 2^{Z}$ and $z \in c_{Z}(A)$. If $z \in X \backslash\{x\}$, then, by (3) in Definition 3, $\pi(z)=z \in c_{X}(\pi(A))$. On the other hand, if $z \in Y$, then, by (3), $\pi(z)=x \in c_{X}(\pi(A))$, and, since in this case $\pi(z)=x$, we get again $\pi(z) \in c_{X}(\pi(A))$, as claimed.

Now let $A, B \in \Omega_{Z}$ and $z_{0}, z_{1} \in Z$ be such that $A \subseteq B, z_{0}, z_{1} \in c_{Z}(A)$, and $z_{1} \in c_{Z}(B)$ hold. We shall show that $z_{0} \in c_{Z}(B)$. To begin with, observe that, since axiom $(\beta)$ holds for $\left(X, c_{X}\right)$, and we have $\pi\left(z_{0}\right), \pi\left(z_{1}\right) \in c_{X}(\pi(A)), \pi\left(z_{1}\right) \in c_{X}(\pi(B))$ (by Lemma 14), and $\pi(A) \subseteq \pi(B)$, then we deduce $\pi\left(z_{0}\right) \in c_{X}(\pi(B))$.

If $\pi\left(z_{0}\right)=z_{0}$, then $z_{0} \in c_{X}(\pi(B)) \backslash\{x\} \subseteq c_{Z}(B)$, so that $z_{0} \in c_{Z}(B)$, as claimed. On the other hand, if $\pi\left(z_{0}\right) \neq z_{0}$, then $\pi\left(z_{0}\right)=x$ and $z_{0} \in Y$. Thus, since $x \in c_{X}(\pi(B))$, we have $c_{Z}(B) \cap Y=c_{Y}(B \cap Y)$, thus proving $z_{0} \in c_{Z}(B)$ is equivalent to showing that $z_{0} \in c_{Y}(B \cap Y)$. As $z_{0} \in c_{Z}(A)$ and $A \subseteq B$, we have $z_{0} \in B$. Recalling that $z_{0} \in Y$, it follows $z_{0} \in B \cap Y$. If $c_{Y}$ is the identity map, then $z_{0} \in c_{Y}(B \cap Y)$ holds. Otherwise, $c_{Y}$ is not the identity map, hence, by (ii), $x$ must be a repellent point of $\left(X, c_{X}\right)$. Since $x \in c_{X}(\pi(B))$, we have $c_{Z}(B)=\left(c_{X}(\pi(B)) \backslash\{x\}\right) \cup c_{Y}(B \cap Y)=c_{Y}(B \cap Y)$. Further, $z_{1} \in c_{Z}(B)$ implies $z_{1} \in c_{Y}(B \cap Y)$. In addition, since $z_{1} \in c_{Z}(A) \cap Y$, then $z_{1} \in c_{Y}(A \cap Y)$. Likewise, since $z_{0} \in c_{Z}(A) \cap Y$, then $z_{0} \in c_{Y}(A \cap Y)$. Thus, as axiom $(\beta)$ holds for $c_{Y}$ and we have $A \cap Y \subseteq B \cap Y, z_{0}, z_{1} \in c_{Y}(A \cap Y)$, and $z_{1} \in c_{Y}(B \cap Y)$, then $z_{0} \in c_{Y}(B \cap Y)$. In conclusion, we obtain $z_{0} \in c_{Y}(B \cap Y) \subseteq c_{Z}(B)$ when $\pi\left(z_{0}\right) \neq z_{0}$, so that $z_{0} \in c_{Z}(B)$ holds in all cases. This proves that (ii) implies (i), thus completing the proof of Theorem 5 .

\section{Section 4 (characterizing resolvability)}

Proof of Theorem 6: $\quad$ Let $A \in 2^{Z}$. We prove $c(A)=c_{Z}(A)$, where $c_{Z}: 2^{Z} \rightarrow 2^{Z}$ is the map 


$$
c_{Z}(A):= \begin{cases}\left(c_{X}(\pi(A)) \backslash\{x\}\right) \cup c(A \cap Y) & \text { if } x \in c_{X}(\pi(A)) \\ c_{X}(\pi(A)) & \text { otherwise }\end{cases}
$$

Thus, the function $c_{Z}$ is the choice correspondence of the one-point resolution of $\left(X, c_{X}\right)$ at $x$ into $\left(Y, c \uparrow_{Y}\right)$, and $\pi: Z \rightarrow X$ is the associated projection.

First, let $x \in \pi(A)$, i.e., $A \cap Y=\emptyset$. By the definition of $c_{X}$ (see Definition 9), we obtain $c_{Z}(A)=c_{X}(\pi(A))=c_{X}(A)=c(A), \quad$ as $\quad$ claimed. Next, suppose $x \in \pi(A)$, hence $A \cap Y \neq \emptyset$. From $\pi(A) \backslash\{x\}=A \backslash Y$, it follows $c_{X}(\pi(A))=\pi(c((\pi(A) \backslash\{x\}) \cup Y))=\pi(c(A \cup Y))$ and also $x \in c_{X}(\pi(A)) \Leftrightarrow x \in \pi(c(A \cup Y)) \Leftrightarrow c(A \cup Y) \cap Y \neq \emptyset$. Thus we obtain

$$
\begin{aligned}
c_{Z}(A) & = \begin{cases}\left(c_{X}(\pi(A)) \backslash\{x\}\right) \cup c(A \cap Y) & \text { if } x \in c_{X}(\pi(A)) \\
c_{X}(\pi(A)) & \text { if } x \notin c_{X}(\pi(A))\end{cases} \\
& = \begin{cases}(\pi(c(A \cup Y)) \backslash\{x\}) \cup c(A \cap Y) & \text { if } c(A \cup Y) \cap Y \neq \emptyset \\
\pi(c(A \cup Y)) & \text { if } c(A \cup Y) \cap Y=\emptyset\end{cases} \\
& = \begin{cases}(c(A \cup Y) \backslash Y) \cup c(A \cap Y) & \text { if } c(A \cup Y) \cap Y \neq \emptyset \\
c(A \cup Y) & \text { if } c(A \cup Y) \cap Y=\emptyset\end{cases} \\
& =c(A),
\end{aligned}
$$

where the last equality follows from a direct application of Lemma 5 . We conclude that the equality $c_{Z}(A)=c(A)$ holds in all cases, and the theorem is fully proved.

Proof of Theorem 7: Let $E$ be a menu in a choice space $(Z, c)$.

(i) $\Rightarrow$ (ii). Assume that $E$ is indiscernible. For each nonempty $E^{\prime} \subseteq E$, property (I2) applied for $A=E^{\prime}$ yields $c\left(E^{\prime}\right)=E^{\prime}$. Thus, the subchoice $c \uparrow_{E}$ is the identity. To complete the proof, we show that $E$ is shrinkable. Property (S2) readily follows from property (I2), since $c \uparrow_{E}$ is the identity. Property (S1) is implied by property (I1): indeed,

$$
\begin{aligned}
& A \cap E \neq \emptyset \quad \Longrightarrow \quad c(A)=c(A \cup E) \cap A \\
& \Longrightarrow \quad c(A) \backslash E=(c(A \cup E) \cap A) \backslash E \quad \cup \quad(c(A \cup E) \backslash(A \cup E)) \\
& \Longrightarrow \quad c(A) \backslash E=(c(A \cup E) \cap A) \backslash E \quad \cup \quad(c(A \cup E) \backslash A) \backslash E \\
& \Longrightarrow \quad c(A) \backslash E=((c(A \cup E) \cap A) \cup(c(A \cup E) \cap(Z \backslash A))) \backslash E \\
& \Longrightarrow \quad c(A) \backslash E=c(A \cup E) \backslash E \text {. }
\end{aligned}
$$

Finally, we show that (S3) is implied by (I1) and (I2). Suppose $A \cap E \neq \emptyset$. Property (I1) gives us $c(A) \cap E=(c(A \cup E) \cap A) \cap E$, hence $c(A \cup E) \cap E=\emptyset \quad$ implies $\quad c(A) \cap E=(c(A \cup E) \cap A) \cap E=\emptyset$. For the reverse implication, suppose $c(A \cup E) \cap E \neq \emptyset$. By applying (I2) to $A \cup E$ (in place of $A$, since $A \cup E$ satisfies the hypothesis $c(A \cup E) \cap E \neq \emptyset$ ), we get $c(A \cup E) \cap E=(A \cup E) \cap E=E$. Now condition (I1) and the hypothesis readily yield $c(A) \cap E=(c(A \cup E) \cap A) \cap E=A \cap E \neq \emptyset$, as claimed. 
(ii) $\Rightarrow(i)$. Assume that $E$ is shrinkable and $c \uparrow_{E}$ is the identity (i.e., the equality $c\left(E^{\prime}\right)=E^{\prime}$ holds for each nonempty $E^{\prime} \subseteq E$ ). Condition (I2) is an immediate consequence of (S2), using the fact that $c \uparrow_{E}$ is the identity. To prove condition (I1), suppose $A \in \Omega_{Z}$ is a menu such that $A \cap E \neq \emptyset$. We shall prove that $c(A)=c(A \cup E) \cap A$. By condition (S1), it suffices to prove

$$
c(A) \cap E=c(A \cup E) \cap A \cap E .
$$

If both $c(A) \cap E$ and $c(A \cup E) \cap E$ are empty, then the equality (15) holds trivially, and we are done. Otherwise, at least one of them is nonempty. Condition (S3) yields that both are nonempty, i.e., $c(A) \cap E \neq \emptyset \neq c(A \cup E) \cap E$. It follows that we can apply condition (S2) to both $A$ and $A \cup E$ as follows:

$$
\begin{aligned}
(c(A \cup E) \cap E) \cap A & =c((A \cup E) \cap E) \cap A & & \text { (by (S2) applied to } A \cup E) \\
& =(A \cap E) \cap A & & \text { (since } c \uparrow_{E} \text { is the identity) } \\
& =c(A \cap E) & & \text { (since } c \uparrow_{E} \text { is the identity) } \\
& =c(A) \cap E & & \text { (by (S2) applied to } A)
\end{aligned}
$$

and so (15) holds.

This completes the proof of the equivalence between (i) and (ii).

Finally, the last statement is an immediate consequence of Corollary 4 (characterization of resolvability) and the fact that indiscernibility implies shrinkability.

\section{Section 5 (rationalizability and resolvability)}

Proof of Theorem 8: Let $(Z, c)$ be a choice space, with $|Z| \geq 3$, such that WARP holds for it. By Theorem 'Rationalization by Axioms' part (ii), there is a total preorder $\succsim$ on $Z$ such that the equality $c(A)=\max (A, \succsim)$ holds for any $A \subseteq Z$. We distinguish the following two cases:

Case 1: $\gtrsim$ is a linear order (i.e., the indifference $\sim$ is the diagonal $\Delta(Z)$ of $Z$ ); Case 2: $\gtrsim$ is a total preorder but not a linear order (i.e., $z_{1} \sim z_{2}$ for some distinct $z_{1}, z_{2} \in Z$ ).

In each of the two cases, we shall show that there is a shrinkable menu, thus proving the claim.

Case 1. Observe that, since $>$ is a linear order, we have $|c(A)|=|\max (A,>)|=1$ for any menu $A \in \Omega_{Z}$, that is, $c$ is indeed a choice function. To prove the claim, we shall need the following general fact about asymmetric binary relations: 
Lemma 15 Let $>_{X}$ be a nonempty asymmetric relation on $X$ such that $\max \left(A,>_{X}\right) \neq \emptyset$ for any nonempty $A \subseteq X$. We have:

(i) there is no strictly increasing infinite sequence (that is, there is no sequence $\left(x_{n}\right)_{n \geq 1}$ of elements in $X$ such that $x_{n+1}>_{X} x_{n}$ for all $\left.n \geq 1\right)$;

(ii) there are two consecutive elements (that is, there are $x, y \in X$ such that $x>_{X} y$ and $x>_{X} z>_{X}$ y for no $z \in X$ ).

Proof For (i), assume by contradiction that there is a sequence $\left(x_{n}\right)_{n \geq 1}$ of elements in $X$ such that $x_{n+1}>_{X} x_{n}$ for all $n \geq 1$. If there are distinct $i, j \geq n, i+1<j$, such that $x_{i}=x_{j}$, then we take $i$ and $j$ such that the integer $j-i \geq 2$ is minimal. Then $x_{j}>_{X} x_{j-1}>_{X} \ldots>_{X} x_{i}=x_{j}$ is a cycle (of minimum size), and the set $A=\left\{x_{i}, x_{i+1}, \ldots, x_{j-1}\right\}$ is such that $\max \left(A,>_{X}\right)=\emptyset$, which is impossible. We can thus assume without loss of generality that all elements in the sequence $\left(x_{n}\right)_{n \geq 1}$ are pairwise distinct. Then the set $A=\left\{x_{n}: n \geq 1\right\}$ has no maximal elements with respect to $>_{X}$, again a contradiction.

For (ii), take $x, y \in X$ such that $x>_{X} y$, which exist since $>_{X}$ is nonempty by hypothesis. If $x$ and $y$ are consecutive, then we are done. Otherwise, there is $x_{1} \in X$ such that $x>_{X} x_{1}>_{X} y$. If $x, x_{1}$ are consecutive, again we are done. Otherwise, there is $x_{2} \in X$ such that $x>_{X} x_{2}>_{X} x_{1}$. By iterating this argument, either there are two consecutive points, or there is a strictly increasing sequence $\left(x_{n}\right)_{n \geq 1}$ such that $x>_{X} \ldots x_{n+1}>_{X} x_{n}>_{X} \ldots>_{X} \ldots x_{1}$. Since the latter fact is impossible by part (i), there must be two consecutive points. This completes the proof of Lemma 15.

Since the asymmetric part $>$ of the total preorder $\gtrsim$ on $Z$ satisfies the hypothesis $^{27}$ of Lemma 15, by part (ii) there are two consecutive items $z_{1}, z_{2} \in Z$ such that $z_{1}>z_{2}$. We claim that the (proper) menu $E=\left\{z_{1}, z_{2}\right\}$ is shrinkable: by Corollary 4 , this will prove the result in Case 1 . To prove the claim, we check that (S1)-(S3) hold for $E$.

For (S1), suppose that $A \in \Omega_{Z}$ is such that $A \cap E \neq \emptyset$. We prove $c(A) \backslash E=c(A \cup E) \backslash E$ by showing the two inclusions. For the first inclusion, suppose $z \in c(A) \backslash E$, i.e., $z \in \max (A,>)$ and $z \neq z_{1}, z_{2}$. Since $A \cap\left\{z_{1}, z_{2}\right\} \neq \emptyset$, $z_{1}>z_{2}$ with $z_{1}$ and $z_{2}$ consecutive, and $>$ is a complete transitive relation, it follows that $z>z_{1}$ and $z>z_{2}$. Thus, $z \in \max (A \cup E) \backslash E=c(A \cup E) \backslash E$, as claimed. The reverse inclusion is immediate. This proves (S1).

For (S2), suppose $c(A) \cap E \neq \emptyset$. Since $z_{1}>z_{2}$, we may only have either $c(A) \cap E=\left\{z_{1}\right\}$ (if $z_{1} \in A$ ) or $c(A) \cap E=\left\{z_{2}\right\}$ (if $z_{2} \in A$ and $z_{1} \notin A$ ). In both cases, the equality $c(A) \cap E=c(A \cap E)$ holds. This proves (S2).

For (S3), suppose $A \cap E \neq \emptyset$. First assume $c(A \cup E) \cap E \neq \emptyset$. The hypothesis yields that we may have either (i) $c(A \cup E) \cap E=\left\{z_{1}\right\}$ (if $z_{1} \in A$ ), or (ii) $c(A \cup E) \cap E=\left\{z_{2}\right\}$ (if $z_{2} \in A$ and $\left.z_{1} \notin A\right)$. Since $c(A) \cap E=\max (A,>) \cap E=\left\{z_{1}\right\}$ in case (i), and $c(A) \cap E=\max (A,>) \cap E=\left\{z_{2}\right\}$ in case (ii), the forward

$\overline{27}$ Notice that $>$ is nonempty, because $|Z| \geq 3$, and $\gtrsim$ is complete and antisymmetric. 
implication holds. For the reverse implication, assume $c(A) \cap E \neq \emptyset$. Again, we may have either (i) $c(A) \cap E=\left\{z_{1}\right\}$ (if $z_{1} \in A$ ), or (ii) $c(A) \cap E=\left\{z_{2}\right\}$ (if $z_{2} \in A$ and $\left.z_{1} \notin A\right)$. In both cases, we get $c(A \cup E) \cap E=\left\{z_{1}\right\}$, as claimed.

Case 2. In this case, there are two distinct items $z_{1}, z_{2} \in Z$ such that $z_{1} \sim z_{2}$. We claim that the proper menu $E=\left\{z_{1}, z_{2}\right\}$ is indiscernible: ${ }^{28}$ by Theorem 7 , this will prove the result in Case 2. To prove the claim, we check that (I1) and (I2) hold for $E$. Observe that, since $\gtrsim$ is a total preorder, we have, for all $z \in Z$,

(1) $z>z_{1}$ if and only if $z>z_{2}$,

(2) $z_{1}>z$ if and only if $z_{2}>z$,

(3) $z \sim z_{1}$ if and only if $z \sim z_{2}$.

Finally, we show that (I1)-(I2) hold for $E$. Let $A \in \Omega_{Z}$. For (I1), suppose $A \cap E \neq \emptyset$. There are three subcases: (i) $A \cap E=\left\{z_{1}\right\}$; (ii) $A \cap E=\left\{z_{2}\right\}$; (iii) $A \cap E=\left\{z_{1}, z_{2}\right\}$. In subcase (i), if $z_{1} \in c(A)$, then properties (1)-(3) yield $c(A \cup E) \cap A=\left(c(A) \cup\left\{z_{2}\right\}\right) \cap A=c(A)$, as claimed. Subcase (ii) can be handled similarly to case (i). For case (iii), we have $A \cup E=A$, whence $c(A \cup E) \cap A=c(A) \cap A=c(A)$, again as claimed.

For (I2), assume that $c(A) \cap E \neq \emptyset$. As for (I1), we deal separately with the three possible subcases, namely: (i) $c(A) \cap E=\left\{z_{1}\right\}$; (ii) $c(A) \cap E=\left\{z_{2}\right\}$; (iii) $c(A) \cap E=\left\{z_{1}, z_{2}\right\}$. In subcase (i), properties (1)-(3) readily yield that $A \cap E=\left\{z_{1}\right\}$, as claimed. Subcase (ii) is handled similarly, whereas subcase (iii) is trivial.

This completes the proof of Theorem 8 .

\section{References}

Aizerman MA, Malishevski AV (1981) General theory of best variants choice. IEEE Trans Autom Control 26(5):1030-1041

Apesteguía J, Ballester MA (2013) Choice by sequential procedures. Games Econ Behav 77:90-99

Arrow KJ (1959) Rational choice functions and orderings. Economica 26:121-127

$\mathrm{Au}$ PH, Kawai K (2011) Sequentially rationalizable choice with transitive rationales. Games Econ Behav 73(2):608-614

Bang-Jensen J, Gutin G (2001) Digraphs. Springer monographs in mathematics. Springer, London Blair DH (1974) Possibility theorems for non-binary social choice functions. Yale University, Mimeo

Cantone D, Giarlotta A, Greco S, Watson S (2016) (m,n)-rationalizable choices. J Math Psychol 73:12-27

Cantone D, Giarlotta A, Watson S (2019) Congruence relations on a choice space. Soc Choice Welfare 52:247-294

Cantone D, Doignon J-P, Giarlotta A, Watson S (2020a) Resolutions of convex geometries. University of Catania, Mimeo

Cantone D, Giarlotta A, Watson S (2020b) Understanding choice behavior: rationalizability vs resolvability. University of Catania, Mimeo

\footnotetext{
28 Since being indiscernible is stronger than being shrinkable, we have some leverage in extending the proof to more general types of preorders: see Cantone et al. (2020).
} 
Chambers CP, Echenique F (2016) Revealed preference theory (Econometric Society Monographs). Cambridge University Press, Cambridge

Cherepanov V, Feddersen T, Sandroni A (2013) Rationalization. Theor Econ 8:775-800

Chernoff H (1954) Rational selection of decision functions. Econometrica 22:422-443

Dekel L, Lipman B, Rustichini A (2001) Representing preferences with a unique subjective state space. Econometrica 49:891-934

Dilworth RP (1940) Lattices with unique irreducible decompositions. Ann Math 41(4):771-777

Edelman PH (1980) Meet-distributive lattices and the anti-exchange closure. Algebra Univ 10(1):290-299

Edelman PH, Jamison R (1985) The theory of convex geometries. Geom Dedicata 19(3):247-270

Eliaz K, Ok EA (2006) Indifference or indecisiveness? Choice-theoretic foundations of incomplete preferences. Games Econ Behav 56:61-86

Falmagne J-C, Doignon J-P (2011) Learning spaces. Springer, Berlin

Fedorčuk VV (1968) Bicompacta with noncoinciding dimensionalities. Soviet Math Doklady 9(5):1148-1150

Gallai T (1967) Transitiv orientierbare graphen. Acta Mathematica Academiae Scientiarum Hungaricae 18(1-2):25-66

García-Sanz M, Alcantud JCR (2015) Sequential rationalization of multivalued choice. Math Soc Sci 74:29-33

Giarlotta A, Watson S (2014) The pseudo-transitivity of preference relations: strict and weak $(m, n)$-Ferrers properties. J Math Psychol 58:45-54

Giarlotta A, Watson S (2017) Necessary and possible indifferences. J Math Psychol 81:98-109

Giarlotta A, Watson S (2018) Strict ( $m, 1)$-Ferrers properties. J Math Psychol 82:84-96

Gul L, Pesendorfer W (2001) Temptation and self-control. Econometrica 69:1403-1435

Herzberger H (1973) Ordinal preference and rational choice. Econometrica 41(2):187-237

Houthakker HS (1950) Revealed preference and the utility function. Economica 17:159-174

Jamison RE (1980) Copoints in antimatroids. In: Proceedings of the eleventh southeastern conference on combinatorics, graph theory and computing, Vol. II, Congressus Numerantium 29, pp 535-544

Kalai G, Rubinstein A, Spiegler R (2002) Rationalizing choice functions by multiple rationales. Econometrica 70(6):2481-2488

Korte B, Lovász L, Schrader R (1991) Greedoids. Springer, Berlin

Koshevoy GA (1999) Choice functions and abstract convex geometries. Math Soc Sci 38:35-44

Leibniz GW (1966) Logical papers, translated and edited by G. H. R. Parkinson. Clarendon, Oxford

Luce RD (1956) Semiorders and a theory of utility discrimination. Econometrica 24:178-191

Manzini P, Mariotti M (2007) Sequentially rationalizable choice. Am Econ Rev 97:1824-1839

Manzini P, Mariotti M (2012) Choice by lexicographic semiorders. Theor Econ 7:1-23

Masatlioglu Y, Nakajima D (2013) Choice by iterative search. Theor Econ 8:701-728

Moulin H (1985) Choice functions over a finite set: a summary. Soc Choice Welfare 2(2):147-160

Moulin H (1986) Choosing from a tournament. Soc Choice Welfare 3(4):271-291

Plott CR (1973) Path independence, rationality, and social choice. Econometrica 41(6):1075-1091

Richter MK (1966) Revealed preference theory. Econometrica 34:635-645

Samuelson P (1938) A note on the pure theory of consumer's behavior. Economica 5:61-71

Sen A (1971) Choice functions and revealed preferences. Rev Econ Stud 38:307-317

Tyson CJ (2013) Behavioral implications of shortlisting procedures. Soc Choice Welfare 41:941-963

Watson S (1992) The Construction of Topological Spaces: Planks and Resolutions. In: Husek M, van Mill J (eds) Recent progress in general topology. North-Holland, Amsterdam, pp 673-757

Publisher's Note Springer Nature remains neutral with regard to jurisdictional claims in published maps and institutional affiliations. 